UCRL-TR-218829

\title{
Phonon Dispersion Curves Determination in $\delta$-phase Pu-Ga Alloys
}

\section{Final Report \\ LDRD Project 03-ERD-017}

J. Wong, D. Clatterbuck, F. Occelli, D. Farber, A. Schwartz

Collaborators: $M$. Wall, C. Boro (LLNL)

M. Krisch, A. Beraud (ESRF, Grenoble, France)

U.S. Department of Energy

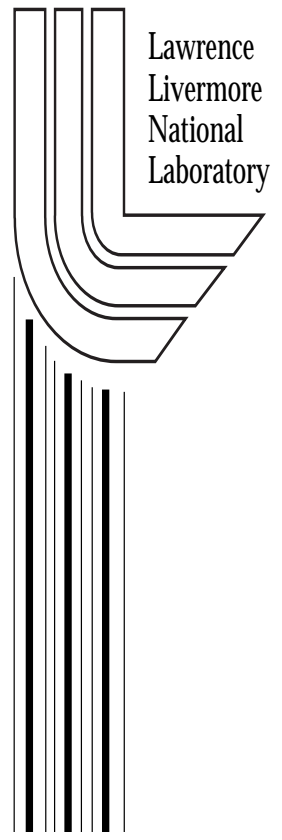
T.C. Chiang, R. Xu, H. Hong, P. Zschack (UIU) N. Tamura, (LBNL)

February 10, 2006 


\section{DISCLAIMER}

This document was prepared as an account of work sponsored by an agency of the United States Government. Neither the United States Government nor the University of California nor any of their employees, makes any warranty, express or implied, or assumes any legal liability or responsibility for the accuracy, completeness, or usefulness of any information, apparatus, product, or process disclosed, or represents that its use would not infringe privately owned rights. Reference herein to any specific commercial product, process, or service by trade name, trademark, manufacturer, or otherwise, does not necessarily constitute or imply its endorsement, recommendation, or favoring by the United States Government or the University of California. The views and opinions of authors expressed herein do not necessarily state or reflect those of the United States Government or the University of California, and shall not be used for advertising or product endorsement purposes.

This work was performed under the auspices of the U. S. Department of Energy by the University of California, Lawrence Livermore National Laboratory under Contract No. W-7405-Eng-48. 


\section{Contents}

Page

$\begin{array}{ll}\text { Summary } & 3\end{array}$

1. Introduction and motivation 4

2. Experimental details 5

2.1 Sample preparation 5

$\begin{array}{lll}2.2 & \text { IXS measurements } & 7\end{array}$

2.3 TDS measurements

2.4 Synchrotron beamtime at ESRF and APS 9

3. Results 10

3.1. Inelastic X-ray scattering data 10

3.2. Thermal diffuse scattering data 13

4. Discussion 16

4.1. Born von-Karman analysis 16

4.2 Elastic properties 17

4.3. Phonon density of states, lattice heat capacity and Debye temperature 22

4.4. $\mathrm{T}(\xi \xi \xi)$ branch bending and the $\delta \rightarrow \alpha$ ' transformation 24

4.5 Anomaly in the $\mathrm{T}_{1}(\xi \xi 0)$ branch 28

4.6. Theoretical considerations 28

5. Other accomplishments 29

$\begin{array}{llr}5.1 & \text { Publications } & 29\end{array}$

5.2 Invited talks 30

5.3 Awards 31

$\begin{array}{lll}5.4 & \text { PR writeups } & 31\end{array}$

5.5 Text Book Quotes 31

6. Impact, future work and concluding remarks 32

$\begin{array}{lll}6.1 & \text { Impact } & 32\end{array}$

$\begin{array}{lll}6.2 & \text { Future work } & 32\end{array}$

6.3 Concluding remarks $\quad 32$

$\begin{array}{ll}\text { References } & 35\end{array}$

$\begin{array}{ll}\text { Postscript } & 38\end{array}$ 


\title{
Phonon Dispersion Curves Determination in $\delta$-phase Pu-Ga Alloys Final Report of LDRD Project 03-ERD-017
}

(February 10, 2006)

\author{
Principal Investigator: Joe Wong \\ Co-investigators: $\quad$ David Clatterbuck, Florent Occelli, Dan Farber, Adam Schwartz, \\ Collaborators: $\quad$ Mark Wall, Carl Boro (LLNL), \\ Michael Krisch, Alex Beraud (ESRF, Grenoble) \\ Tai-C. Chiang, Ruqing Xu, Hawoong Hong, Paul Zschack (UIU) \\ N. Tamura (LBNL)
}

Responsible Associate Directors: Bruce Goodwin (DNT); Tomas Diaz de la Rubia (CMS)

\section{Summary}

We have designed and successfully employed a novel microbeam on large grain sample concept to conduct high resolution inelastic x-ray scattering (HRIXS) experiments to map the full phonon dispersion curves of an fcc $\delta$-phase Pu-Ga alloy. This approach obviates experimental difficulties with conventional inelastic neutron scattering due to the high absorption cross section of the common ${ }^{239} \mathrm{Pu}$ isotope and the non-availability of large (mm size) single crystal materials for $\mathrm{Pu}$ and its alloys. A classical Born von-Kármán force constant model was used to model the experimental results, and no less than $4^{\text {th }}$ nearest neighbor interactions had to be included to account for the observation. Several unusual features including, a large elastic anisotropy, a small shear elastic modulus, $\left(\mathrm{C}_{11}-\mathrm{C}_{12}\right) / 2$, a Kohn-like anomaly in the $T_{1}[011]$ branch, and a pronounced softening of the T[111] branch towards the $\mathrm{L}$ point in the Brillouin are found. These features may be related to the phase transitions of plutonium and to strong coupling between the crystal structure and the $5 f$ valence instabilities. Our results represent the first full phonon dispersions ever obtained for any Pu-bearing material, thus ending a 40-year quest for this fundamental data. The phonon data also provide a critical test for theoretical treatments of highly correlated $5 f$ electron systems as exemplified by recent dynamical mean field theory (DMFT) calculations for $\delta$-plutonium. We also conducted thermal diffuse scattering experiments to study the $\mathrm{T}(111)$ dispersion at low temperatures with an attempt to gain insight into bending of the T(111) branch in relationship to the $\delta \rightarrow \alpha^{\prime}$ transformation. 


\section{Introduction and Motivation}

Plutonium $(\mathrm{Pu})$ is certainly the most astonishing member of the actinides, the class of the elements in which the $5 f$ electron shell is progressively filled. Indeed, in the early part of this series (Th, $\mathrm{Pa}, \mathrm{U}$ and $\mathrm{Np}$ ), the $5 f$ electrons contribute to the bonding between atoms. The $5 f$ participation in bonding results in an atomic volume dependence on electron population similar to that of the transition metal series. On the other hand, the heavier members of the actinide series ( $\mathrm{Am}, \mathrm{Cm}$ and beyond) have larger atomic volumes that are almost independent of the $5 f$ electron population. This behavior resembles those of the lanthanide elements; the $5 f$ states are localized and do not participate in the bonding. In $\mathrm{Pu}$, the $5 f$ electrons are "on the edge," 1 and it is this unique $5 f$ configuration that gives this element a host of unusual properties. ${ }^{2,3}$ Since the discovery of Pu in 1941, the element's eccentricities have both awed and perplexed researchers. Although the element's complexity and unpredictability have rendered it a challenge to study, scientists persist because of the need to understand and predict plutonium's behavior under various temperatures and pressures and to determine how the element's physical properties might vary over time. Understanding its properties is indeed critical for the safe handling, use, and long-term storage of this highly toxic and radioactive material. ${ }^{4}$

The most notably unusual property of $\mathrm{Pu}$ is perhaps the presence of 5 solid-state allotropic phase transformations in the pure element. The $\alpha$ phase, which is stable from low temperature to $122^{\circ} \mathrm{C}$, transforms successively to $\beta \rightarrow \gamma \rightarrow \delta \rightarrow \delta^{\prime} \rightarrow \varepsilon \rightarrow$ liquid with increasing temperature ${ }^{3}$ accompanied by large volume expansions and collapses along the way to the melt. The pure metal melts at a relatively low temperature $\sim 640^{\circ} \mathrm{C}$ to yield a liquid which is higher in density than that of the solid from which it melts. The fcc (face-centered-cubic) $\delta$ phase is the equilibrium phase in the range $319-451^{\circ} \mathrm{C}$, and has a density of $15.92 \mathrm{~g} / \mathrm{cc}$. This high temperature $\delta$ phase can be stabilized or more correctly retained at room temperature and below by alloying with small amounts of Group III metals such as $\mathrm{Al}$ or Ga. ${ }^{5}$ In doing so, the metastable $\delta$-phase field is expanded from high temperature to room temperature and below. Moreover, alloys of $\delta \mathrm{Pu}-\mathrm{Ga}$ are known to transform directly to the $\alpha$ or, more correctly, $\alpha$ ' monoclinic phase, bypassing both the $\gamma$ and $\beta$ phases via a martensitic-like transformation ${ }^{3,5,6}$ which is displacive in nature and diffusionless. In many systems that undergo a martensitic phase transformation, such as NiTi,

N.B.: $\alpha^{\prime}$ is used to denote a monoclinic phase of $\mathrm{Pu}-\mathrm{Ga}$ alloy transformed from $\delta \mathrm{Pu}-\mathrm{Ga}$ at low temperature. $\alpha$ is reserved for a pure $\mathrm{Pu}$ phase below $123{ }^{\circ} \mathrm{C}$. 
$\mathrm{NiAl}$, and $\mathrm{Ni}_{2} \mathrm{MnGa}$, significant phonon softening is observed in the transverse acoustic mode ${ }^{7}$.

Phonon dispersion curves (PDC) are very fundamental to the derivation of material properties such as force constants, sound velocities, elastic and thermodynamic properties, and to the study phase stability, electron-phonon coupling (charge-density waves), structural relaxation, etc. PDCs are key experimental data to the understanding of basic and often time intriguing properties of Pu materials, their phase changes and associated phase stability. Yet, PDC measurements on Pu and its alloys with conventional inelastic neutron scattering (INS) are hindered by the facts that (i) $\mathrm{Pu}$ has a high neutron absorption cross section for the most common ${ }^{239} \mathrm{Pu}$ isotope, and (ii) the large single crystals of $\mathrm{Pu}$ (a few $\mathrm{mm}$ cube) required for INS experiments are not available. These limitations have recently been overcome experimentally by the application of the high resolution inelastic x-ray scattering (HRIXS) technique, combined with a microbeam on large grain sample concept ${ }^{8}$ by focusing a high brightness $\mathrm{x}$-ray beam on large single-crystal domains in a polycrystalline specimen. Furthermore, only recently have theoretical computations of the Pu PDCs begun to overcome the difficulties in treating the $5 f$ electrons accurately within the standard first-principles methods. ${ }^{9-12}$ Thus, until 2003, the PDCs for Pu-bearing systems have remained essentially unknown experimentally ${ }^{8}$ and theoretically. ${ }^{11}$

\section{Experimental Details}

\subsection{Sample preparation}

Our samples were large-grain polycrystalline specimens prepared by a strain-enhanced recrystallization technique ${ }^{13}$ from an fcc $\delta \mathrm{Pu}-\mathrm{Ga}$ alloy containing 2 at. \% Ga. A cylinder of the homogenized alloy, $2.8 \mathrm{~mm}$ diameter by $2 \mathrm{~mm}$ high was first uniaxially compressed by $6 \%$ and annealed at $460^{\circ} \mathrm{C}$ in a vacuum $\left(5 \times 10^{-5}\right.$ Torr) for 5 days. Two such strain-anneal cycles were employed. The doubly annealed cylinder was then sliced at 45 degrees to the cylinder axis into 3 slices, each $\sim 500 \mu \mathrm{m}$

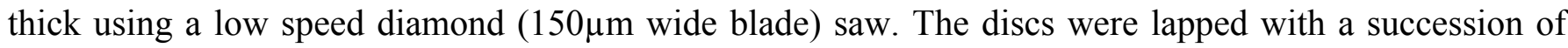
lapping films, 600 grit $\mathrm{SiC}, 30 \mu \mathrm{m}, 12 \mu \mathrm{m}$, and $3 \mu \mathrm{m} \gamma-\mathrm{Al}_{2} \mathrm{O}_{3}$ to a thickness of $\sim 40 \mu \mathrm{m}$. A fine polish with $1 \mu \mathrm{m}$ diamonds followed to remove $\sim 12 \mu \mathrm{m}$ from each side, yielding a thickness of $\sim 16-18 \mu \mathrm{m}$. The samples were then electropolished from both sides using a TEM (transmission electron microscopy) electropolishing system such that a shallow dimple was electrochemically removed from both side of the disc specimens to render a final thickness of $\sim 8-10 \mu \mathrm{m}$. This thickness is about one absorption length for $\delta-\mathrm{Pu}^{14}$ at $21.747 \mathrm{keV}$ allowing for optimal IXS signals in transmission geometry. This procedure produced a microstructure with an average grain size of $\sim 90 \mu \mathrm{m}$ as shown in Fig. 1. The samples were dip 
coated in liquid polyamide and cured at $50^{\circ} \mathrm{C}$ for $1 \mathrm{hr}$. This provided the first containment layer. The polyamide coated $\mathrm{Pu}-\mathrm{Ga}$ foil was then loaded into a leak-proof stainless steel cell in a dry nitrogen atmosphere. The cell contained a pair of kapton windows each $125 \mu \mathrm{m}$ thick which provide a second level of containment and a third metal containment for shipment from Livermore to Greble. ${ }^{15}$

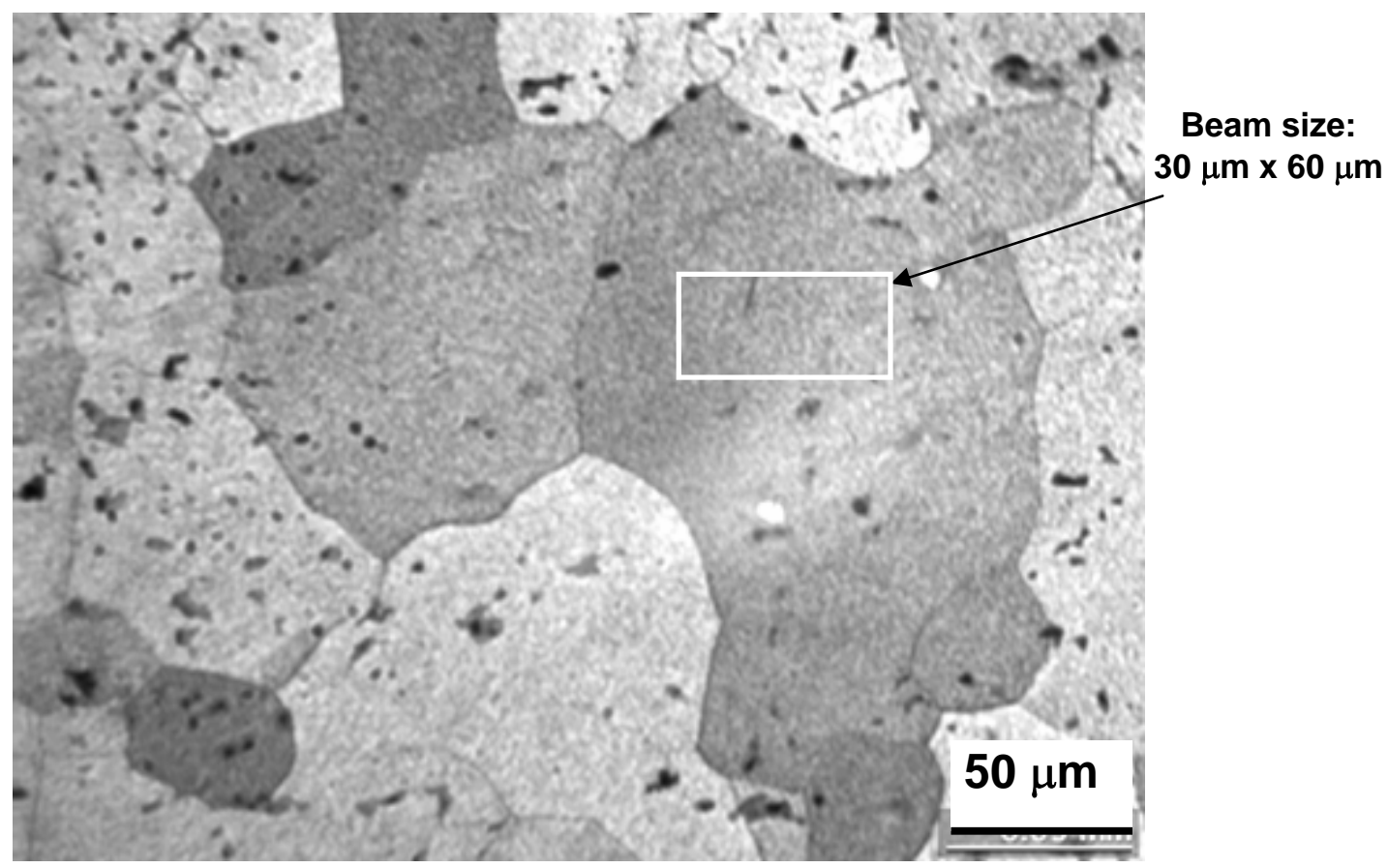

Fig. 1. Optical micrograph showing the large-grain microstructure of the fcc Pu-Ga 2 at. \% alloy used in this study. The white box denotes the footprint on the sample of a $30 \mu \mathrm{m} \times 60 \mu \mathrm{m}$ x-ray beam used to collect the HRIXS phonon spectra.

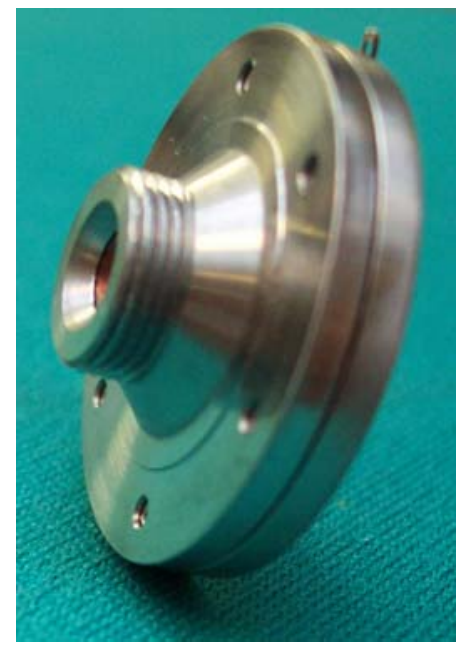

Fig. 2. Sample cell used to hold the Pu-Ga alloy samples in multiple containment. 


\subsection{IXS Measurements}

High resolution IXS experiments were conducted on beamline ID28 at the European Synchrotron Radiation Facility (ESRF) in Grenoble, France. The storage ring was operating at an electron energy of 6 $\mathrm{GeV}$ and injection current of $200 \mathrm{~mA}$. The synchrotron radiation was monochromatized at $21.747 \mathrm{keV}$ utilizing the $\operatorname{Si}\left(\begin{array}{lll}1 & 11 & 11\end{array}\right)$ configuration to yield a total instrumental energy resolution of $1.8 \mathrm{meV}$ fullwidth-at-half-maximum (FWHM) with the dimensions of the focused x-ray beam $30 \mu \mathrm{m}$ horizontal x 60 $\mu \mathrm{m}$ vertical FWHM (see Fig. 1). With this beam, the flux on the sample was measured to be $\sim 3 \times 10^{9}$ photons/s. The scattered photons were energy-analyzed by five crystal analyzers in Rowland circle geometry employing the same reflection order as the high-resolution backscattering monochromator. The momentum transfer, $\mathrm{Q}=2 \mathrm{k}_{0} \sin \left(\theta_{\mathrm{s}} / 2\right)$, where $\mathrm{k}_{0}$ is the wave vector of the incident photons and $\theta_{\mathrm{s}}$ the scattering angle, was selected by rotating the spectrometer arm in the horizontal plane. The momentum resolution was set by slits to $0.4 \mathrm{~nm}^{-1}$. An on-line fluorescent screen, coupled to a CCD camera, allowed us to map and select single-crystal domains in the specimen, and orient them according to the requirements of the scattering geometry for the longitudinal and transverse acoustic phonon branches. Mapping of the single-crystal domains was very time-consuming, but necessary, requiring over $50 \%$ of our usable beam time. With the small beam dimensions, single-crystal domains could be selected with a typical mosaic spread ranging between 0.5 and 1.5 degrees. In the longitudinal geometry, after appropriate momentum transfer corrections ${ }^{16}$ the spectra of all five analyzers could be utilized. This was not possible for the transverse scans, and therefore the spectrum of only one analyzer was utilized. The energy scans were performed by varying the monochromator temperature while the analyzer temperature was kept constant. Conversion from the temperature scale to the energy scale was accomplished by using the following relation: $\Delta \mathrm{E} / \mathrm{E}=\alpha \Delta \mathrm{T}$, where $\alpha=2.58 \times 10^{-6} \mathrm{~K}^{-1}$ is the linear thermal expansion coefficient of $\mathrm{Si}$ at room temperature. Details of the HRIXS instrumentation have been described elsewhere. ${ }^{17}$ All phonon spectra for full phonon dispersions of the Pu-2 at. \% Ga alloy along all the principal symmetry directions (Fig. 4) in this study were collected with the samples at room temperature.

\subsection{TDS Measurements}

The materials studied are Pu-Ga alloys containing 0.6 to $1.2 \mathrm{wt}$. \% of $\mathrm{Ga}$ for stabilizing $\mathrm{Pu}$ in the $\delta$-phase. The sample is $2.8 \mathrm{~mm}$ in diameter and about $15 \mu \mathrm{m}$ thick with grain sizes in the range of 50-100 $\mu \mathrm{m}$ as determined by optical microscopy. The sample was mounted in an aluminum containment chamber 
in the form of a cylinder with a diameter of $70 \mathrm{~mm}$, a height of $90 \mathrm{~mm}$, and a wall thickness of $0.8 \mathrm{~mm}$ (Fig. 3). These dimensions were chosen for an $18 \mathrm{keV}$ beam to yield a desired maximum momentum transfer of $\sim 4 \AA^{-1}$ (the lattice constant of the material is $4.621 \AA$ ). An interior mounting plate and an

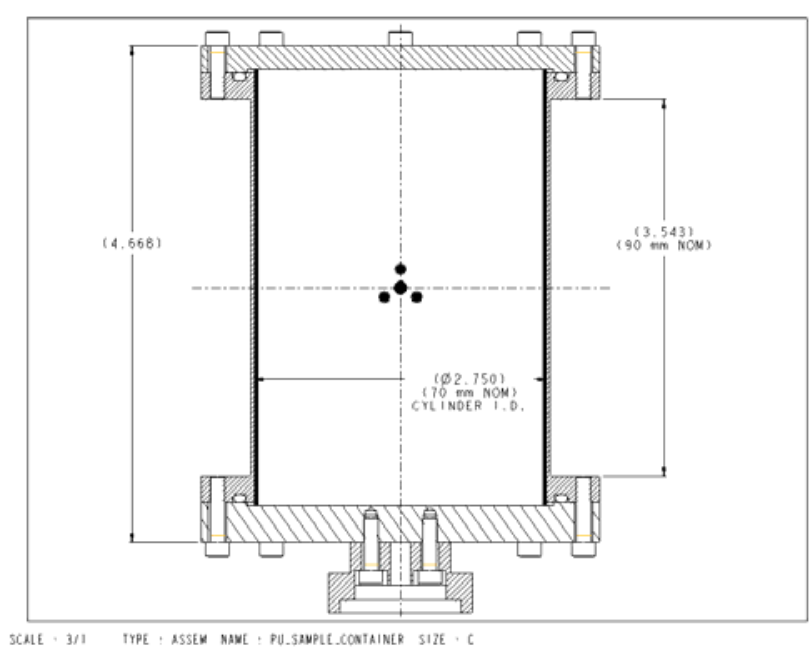

(a)

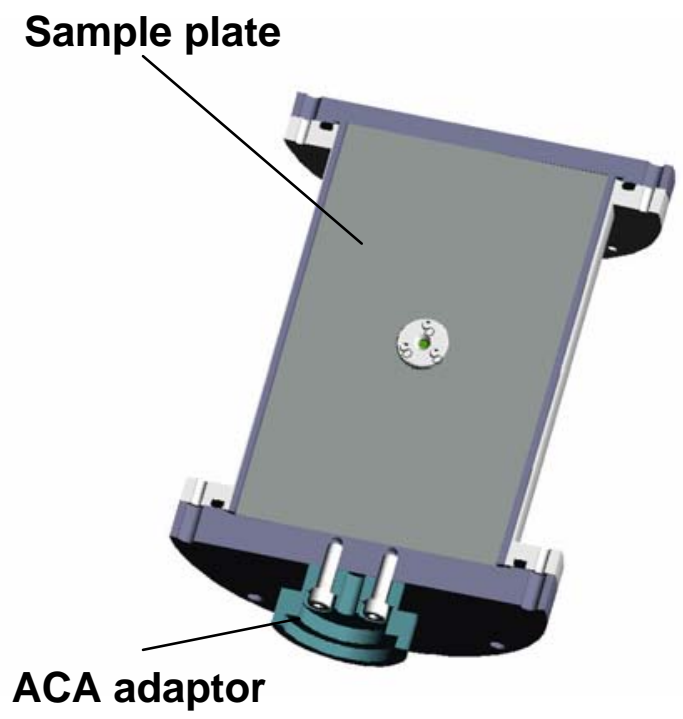

(b)

Fig. 3 (a) Cross sectional view of the primary Pu sample chamber for TDS experiments; (b) semi-solid perspective view of the same

external lead-disk beam stop were used, respectively, to prevent scattering from the upstream and downstream aluminum containment walls from reaching the detector. The containment chamber was needed both for safety reasons ( $\mathrm{Pu}$ is highly radioactive and toxic) and for preserving sample integrity ( $\mathrm{Pu}$ burns in air spontaneously and must be kept under an inert atmosphere at all times).

The TDS measurements were performed using the undulator beamline of UNICAT (University, National Laboratory, and Industry Collaborative Access Team) at the Advanced Photon Source (APS), Argonne National Laboratory, with the APS storage ring operating at $7 \mathrm{GeV}$ and a current of $100 \mathrm{~mA}$. The x-ray energy was set to $18.000 \mathrm{keV}$ using a double $\mathrm{Si}(111)$ crystal monochromator. This energy is $\sim 50 \mathrm{eV}$ below the $\mathrm{Pu} \mathrm{L} \mathrm{L}_{3}$ edge, and the sample transmission is near a maximum with an absorption length of $\sim 14 \mu \mathrm{m}$. The incident beam was focused to $\sim 100 \mu \mathrm{m} \times 260 \mu \mathrm{m}$, and passed successively through a $0.5 \mathrm{~mm} \times 0.5 \mathrm{~mm}$ slit assembly and a pinhole to yield a $25-\mu \mathrm{m}$-diameter beam on the sample with an estimated flux of $\sim 6 \times 10^{10}$ photons/s. A transmission Laue geometry was employed, and the pattern of the scattered intensity was recorded by a charge-coupled device (CCD) positioned immediately behind the 
sample containment chamber. The exposure times were typically in the range of 5 to $30 \mathrm{~s}$. All measurements were performed with the sample at room temperature. To check the beam alignment and background level, a test was performed first with a $\mathrm{Si}(111)$ wafer sample mounted in the containment chamber. A clean TDS image exhibiting three-fold symmetry identical to that reported in the literature [ Holt et al., Phy. Rev. Lett. 83,3317 (1999)] was obtained with no discernable diffraction lines or spots from the chamber walls or beamline components. Thus, all visible features, including powder rings, in the images to be presented below are generated from the sample itself.

Area survey scans of scattering images were recorded systematically to map the single-crystal domains in the polycrystalline microstructure of the $\mathrm{Pu}-\mathrm{Ga}$ specimen. The procedure consisted of scanning, for sample, a $500 \mu \mathrm{m}$ by $500 \mu \mathrm{m}$ sample area with the $25 \mu \mathrm{m}$ beam using a step size of $50 \mu \mathrm{m}$ to yield a total of 121 scans. Each frame was visually examined to identify single-crystal domains. Of special interest were the (100), (111) and (110) domains with 4-, 3- and 2-fold symmetry, respectively. Five such $500 \mu \mathrm{m}$ x $500 \mu \mathrm{m}$ areas were mapped. Initally, we were successful in locating a number of (111)- and (100)-oriented grains, and later (110) grains on other samples. At each located (hkl) grain, we performed a series of multiple exposures $(60-100)$ with an exposure time of 20 or $30 \mathrm{sec}$ and the CCD operating at $\sim 50 \%$ duty cycle. We also used a 345 MAR detector in the most recent runs in the past two years. Each set of multiple scans was summed to give the final experimental TDS image.

\subsection{Synchrotron Beamtime Award at ESRF, Grenoble and Argonne APS}

We have received a top rating for our beam time proposals for IXS and TDS experiments respectively at both European Synchrotron Radiation Facility (ESRF), Grenoble and the Advanced.Photon Source (APS) at Argonne.

\begin{tabular}{lclc} 
ESRF & \# of 8 hr shifts & APS & \# of 8 hr shifts \\
\hline Feb/03 & 20 & Mar/04 & 17 \\
Mar/03 & 18 & Jul/04 & 16 \\
Feb/04 & $*$ & Dec/04 & 23 \\
Apr/04 & 23 & Aug/05 & 15 \\
Jun/05 & 21 & & \\
\hline
\end{tabular}

* Beamtime was awarded but not taken due to delay of the French authorization for sample shipmen 
Also, with the collaboration of Dr. N. Tamura at the Berkeley ALS, we were successful in securing 20 shifts of 2-bunch beam (low flux) at the ALS on his micro-diffraction beamline (\#7.3.3.3) to perform some micro-Laue mapping of the $\mathrm{Pu}-\mathrm{Ga}$ samples. The overall results indicate that the crystallographic grain size is about $30 \%$ smaller than the metallographic values from optical microscopy.

\section{Results}

\subsection{Inelastic X-ray Scattering Data}

Typical IXS spectra (Fig. 4) are shown for longitudinal acoustic (LA) phonons along the [111] direction at selected reduced momentum transfer values $(\xi \xi \xi)$. At a given $\boldsymbol{q}$ value, the count rate with
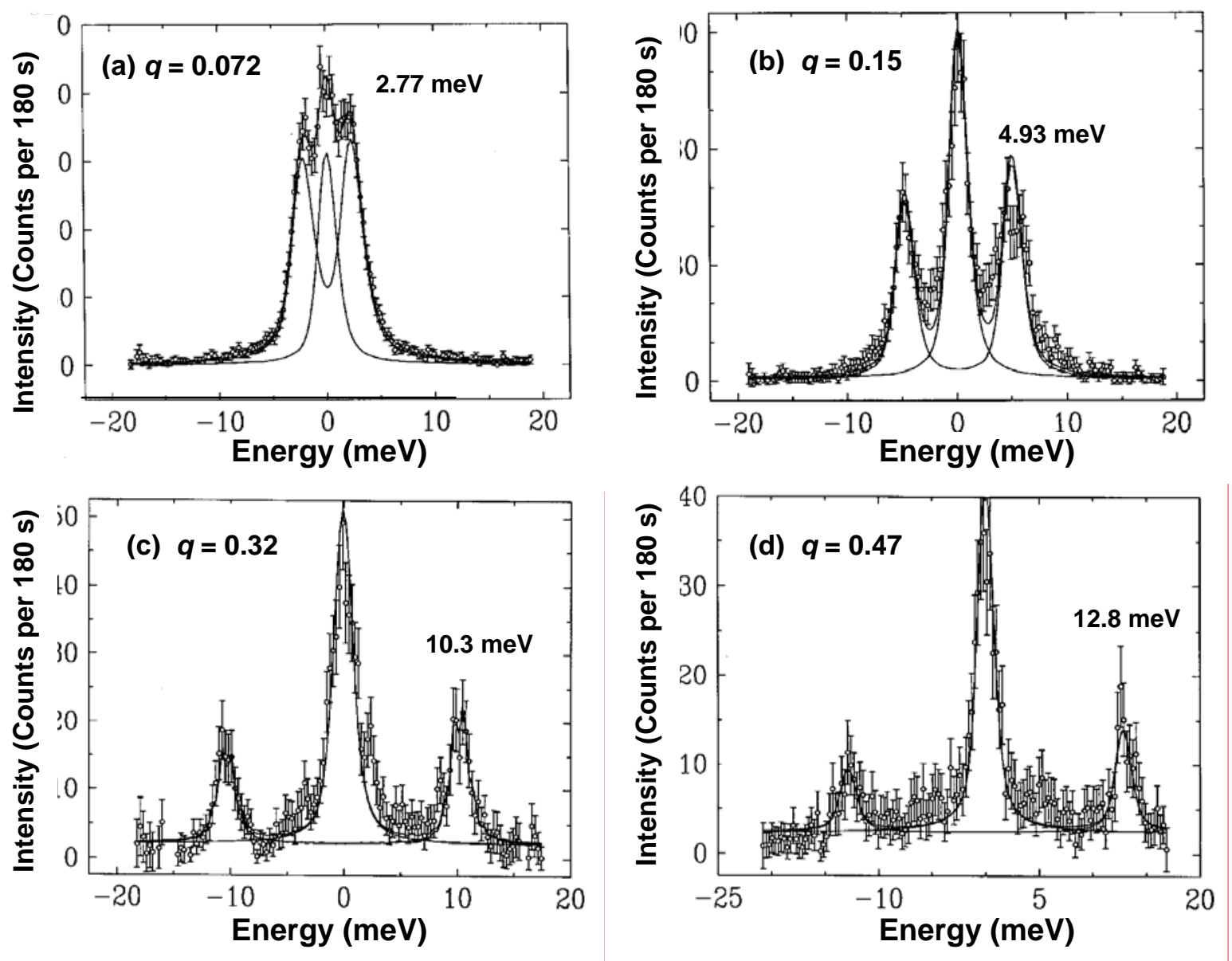

Fig. 4. Representative inelastic $x$-ray scattering spectra for the longitudinal acoustic phonon branch along the [111] direction in a $\delta \mathrm{Pu}-2$ at. \% Ga alloy. The experimental data (points) are shown together with the results of the best fit model (lines) of the phonon and elastic contributions. In each panel, the reduced phonon wave vector, $\xi$, is indicated to the left of the spectra and the phonon energy to the right. 
error bars is plotted versus the energy of the analyzer. Each spectrum in Fig. 3 results from a sum of two individual 4-hr scans. For each scan, the integration time per data point was 90s. The spectra are each characterized by an elastic contribution centered at zero energy and two inelastic contributions, corresponding to the creation (energy-loss, Stokes) and annihilation (energy gain, anti-Stokes) of an acoustic phonon. In order to extract the phonon energies, the spectra were fitted to a model function convoluted with the experimentally determined resolution function, by using a standard $\chi^{2}$ minimization procedure. This function consisted of a pair of Lorentzians for the Stokes and anti-Stokes excitations with their intensity ratio governed by the thermal phonon population factor, and a single Lorentzian for the central Rayleigh line.

The experimental phonon dispersion curves along these symmetry directions are plotted as points in Fig. 5, together with a fit (solid lines) obtained by means of a standard Born-von Kármán (B-vK) force

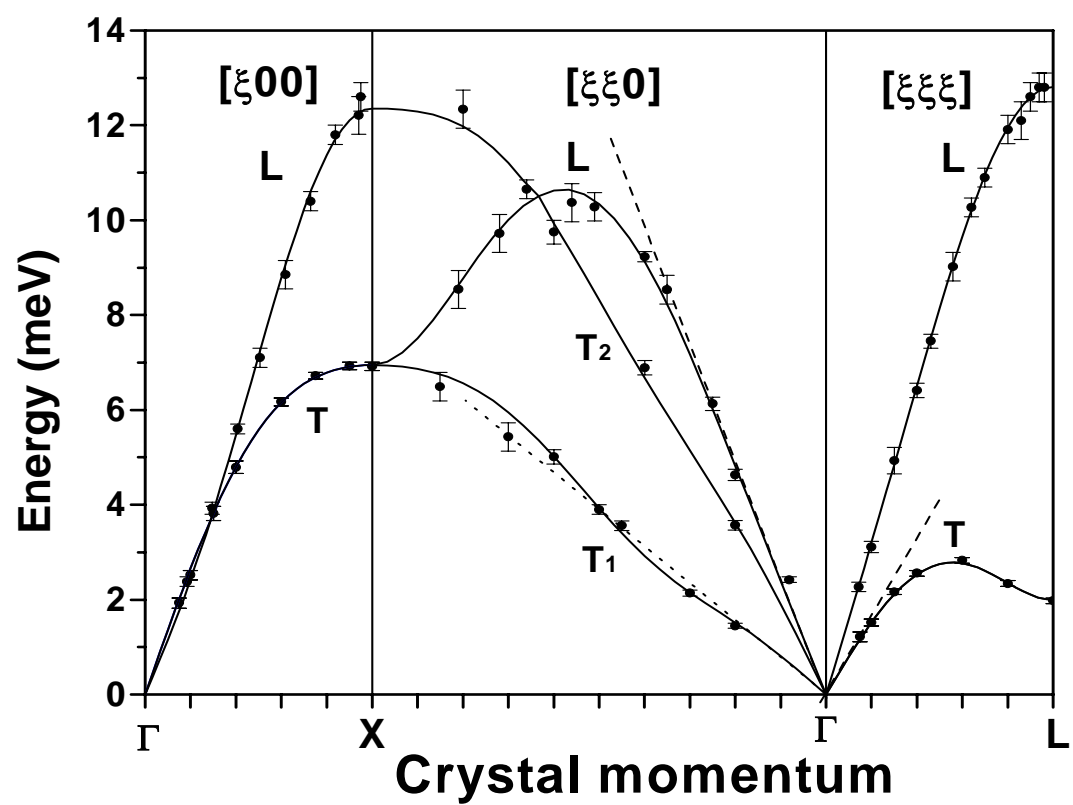

Fig. 5. The phonon dispersion curves of a $\delta$ fcc $\mathrm{Pu}-2$ at. \% Ga alloy at room temperature. The experimental points are plotted as circles, while the solid curves represent a fourth-nearest neighbor (4NN) Born-von Kármán model fit to the experimental data. The lattice parameter of the alloy is $0.4621 \mathrm{~nm}$. Longitudinal and transverse modes are denoted $\mathrm{L}$ and $\mathrm{T}$ respectively The transverse branches along each of the $[\xi 00]$ and $[\xi \xi \xi]$ directions are degenerate. Along the $[\xi \xi 0]$ direction, the two transverse branches are distinct: $\mathrm{T}_{1}[011]$ polarized along $<01 \overline{1}>$ and $\mathrm{T}_{2}[011]$ polarized along $<001>$. Note the pronounced bending of the $\mathrm{T}[\xi \xi \xi]$ branch near the $\mathrm{L}$ point. The dotted lines are linear fits at low qs at the $\Gamma$ point at the selected branches 
Table 1. Normal mode phonon energies for the symmetry branches in a $\delta \mathrm{Pu}-2$ at.\% Ga alloy at room temperature.

\begin{tabular}{|c|c|}
\hline \multicolumn{2}{|c|}{$L(0,0, \xi)$} \\
\hline$\xi$ & meV \\
\hline & 0 \\
\hline 0.185 & $2.38 \pm 0.3$ \\
\hline 0.296 & $4.23 \pm 0.2$ \\
\hline 0.407 & $6.0 \pm 0.2$ \\
\hline 0.506 & $7.45 \pm 0.2$ \\
\hline 0.619 & $8.85 \pm 0.3$ \\
\hline 0.729 & $10.4 \pm 0.2$ \\
\hline 0.838 & $11.8 \pm 0.2$ \\
\hline 0.951 & $12.6 \pm 0.3$ \\
\hline 0.941 & $12.21 \pm 0$. \\
\hline
\end{tabular}

$\mathbf{L}(\mathbf{0}, \xi, \xi)$

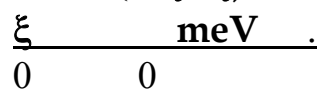

$0.1 \quad 2.42 \pm 0.06$

$0.2 \quad 4.63 \pm 0.12$

$0.25 \quad 6.13 \pm 0.14$

$0.35 \quad 8.53 \pm 0.3$

$0.4 \quad 9.23 \pm 0.11$

$0.51 \quad 10.28 \pm 0.3$

$0.56 \quad 10.37 \pm 0.4$

$0.66 \quad 10.65 \pm 0.2$

$0.72 \quad 9.72 \pm 0.4$

$0.81 \quad 8.54 \pm 0.4$

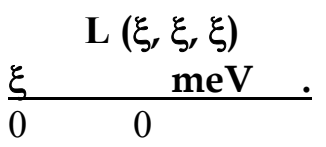

$0.072 \quad 2.27 \pm 0.1$

$0.1 \quad 3.11 \pm 0.12$

$0.15 \quad 4.93 \pm 0.28$

$0.2 \quad 6.41 \pm 0.15$

$0.23 \quad 7.45 \pm 0.15$

$0.28 \quad 9.02 \pm 0.3$

$0.32 \quad 10.27 \pm 0.2$

$0.35 \quad 10.9 \pm 0.2$

$0.4 \quad 11.91 \pm 0.3$

$0.43 \quad 12.1 \pm 0.4$

$0.45 \quad 12.6 \pm 0.3$

$0.47 \quad 12.8 \pm 0.3$

$0.48 \quad 12.8 \pm 0.3$
$\mathbf{T}(\mathbf{0}, \mathbf{0}, \xi)$

\begin{tabular}{ll}
$\xi$ & \multicolumn{1}{c}{$\mathbf{m e V}$} \\
\hline 0 & 0 \\
0.15 & $1.93 \pm 0.1$ \\
0.2 & $2.52 \pm 0.1$ \\
0.3 & $3.82 \pm 0.15$ \\
0.4 & $4.79 \pm 0.13$ \\
0.6 & $6.17 \pm 0.08$ \\
0.75 & $6.72 \pm 0.07$ \\
0.9 & $6.93 \pm 0.08$ \\
1 & $6.92 \pm 0.09$
\end{tabular}

\begin{tabular}{ll} 
& $\begin{array}{c}\left.\mathbf{T}_{\mathbf{2}} \mathbf{( 0 ,}, \boldsymbol{\xi}, \boldsymbol{\xi}\right) \\
\mathbf{m e V}\end{array}$ \\
\hline 0 & 0 \\
0.2 & $3.57 \pm 0.1$ \\
0.4 & $6.89 \pm 0.15$ \\
0.6 & $9.75 \pm 0.25$ \\
0.8 & $12.34 \pm 0.4$
\end{tabular}

\begin{tabular}{|c|c|}
\hline \multicolumn{2}{|c|}{$\mathbf{T}_{1}(0, \xi, \xi)$} \\
\hline$\xi$ & meV \\
\hline 0 & 0 \\
\hline 0.2 & $1.45 \pm 0.05$ \\
\hline 0.3 & $2.14 \pm 0.0$ \\
\hline 0.45 & $3.56 \pm 0.1$ \\
\hline 0.5 & $3.9 \pm 0.1$ \\
\hline 0.6 & $5.01 \pm 0.15$ \\
\hline 0.7 & $5.43 \pm 0.3$ \\
\hline 0.85 & $6.49 \pm 0.3$ \\
\hline
\end{tabular}

$\mathbf{T}(\xi, \xi, \xi)$

\begin{tabular}{ll}
$\xi$ & \multicolumn{1}{c}{$\mathbf{m e V}$} \\
0 & 0 \\
0.075 & $1.22 \pm 0.1$ \\
0.1 & $1.52 \pm 0.07$ \\
0.15 & $2.164 \pm 0.06$ \\
0.2 & $2.56 \pm 0.06$ \\
0.3 & $2.83 \pm 0.06$ \\
0.4 & $2.34 \pm 0.06$ \\
0.5 & $1.98 \pm 0.0$
\end{tabular}


constant model. ${ }^{18}$ An adequate fit to the experimental dispersions is obtained with this method if interactions up to the fourth nearest neighbor are included. Along the $[\xi 00]$ and $[\xi \xi \xi]$ directions, the transverse modes are degenerate and $\zeta$, the direction of vibration of the single atom in the unit cell, may lie in any direction normal to $\boldsymbol{q}$. Along the $[\xi \xi 0]$ direction, the two transverse modes are distinct with $\zeta$ lying in the [01 $\overline{1}]$ and [001] directions for $\mathrm{T}_{1}$ and $\mathrm{T}_{2}$ respectively.

Values of the phonon energies for the three main symmetry directions of the $\delta \mathrm{Pu}-\mathrm{Ga}$ fcc structure, with estimates of overall errors resulting from the $\chi^{2}$ minimization algorithm, are given in Table 1 . Three unusual features in the PDCs of $\delta \mathrm{Pu}-\mathrm{Ga}$ alloy are noteworthy: (a) similarity in the slopes of the LA( $(\xi 00)$ and $\mathrm{TA}\left(\xi_{00)}\right)$ branches, (b) a kink in the $\mathrm{T}_{1}\left(\xi \xi_{0}\right)$ branch suggestive of a Kohn anomaly ${ }^{19}$ and (c) a pronounced bending of the $\mathrm{TA}(\xi \xi \xi)$ branch near the L point. These features will be discussed in detail below.

\subsection{Thermal Diffuse Scattering Data}

Images in Fig. 6(a) are transmission x-ray TDS images each from a corresponding (111), (110) and (100) grain. These images are obtained from a summation of 60 exposures, each for 30 s. Ignoring the Debye rings, the TDS pattern clearly exhibits 3-, 2- and 4-fold symmetry characteristic of the respective directions. As is known from previous work, TDS patterns arise from x-ray scattering from thermally populated phonons. Each point in the image corresponds to a unique momentum transfer. Regions in kspace close to the reciprocal lattice points as defined by the Ewald sphere give rise to intense spots due to a high phonon population. Streaks connecting the intense spots are generally associated with high symmetry directions in the Brillouin zone. The image also shows the shadow of the beam stop. Shown in Fig. 6(b), for comparison, are the corresponding calculated TDS patterns based on least-squares fits to the experimental results. Apart from the Debye rings, the computed images closely reproduce the respective experimental patterns. The Debye rings have been indexed and can be shown to derive from microcrystalline $\delta$-Pu-Ga (major phase) and $\mathrm{PuO}$ impurity (minor phase). The presence of these rings suggests that the best Pu-Ga samples currently available to us are not yet of sufficiently high quality for clean, quantitative TDS work. The pattern shown in Fig 6(a), however, indicate that the sample 
$\delta$ Pu-Ga (111)
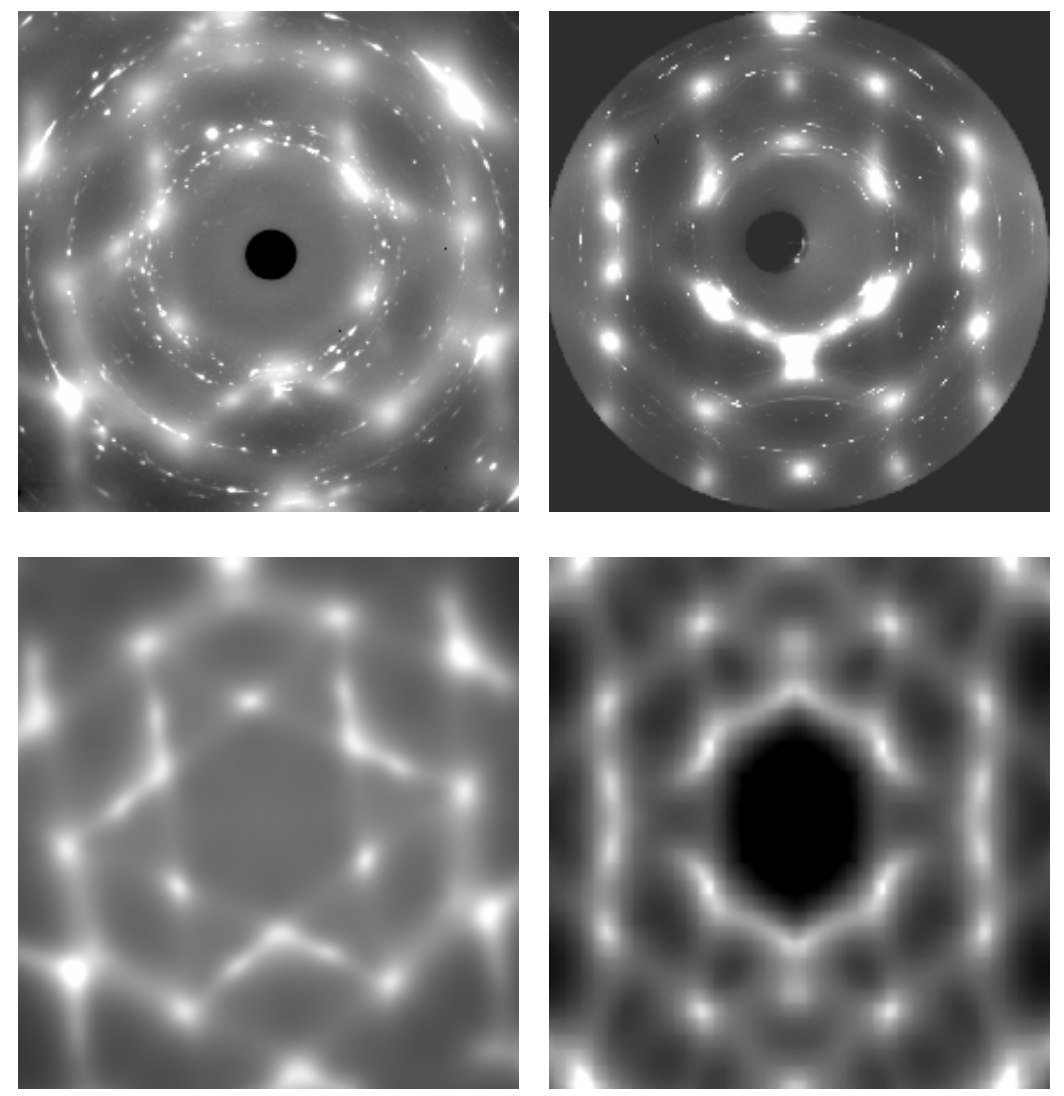

$\delta$ Pu-Ga (100)

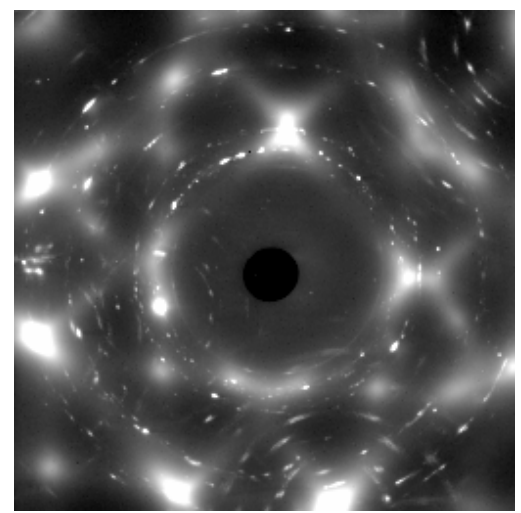

(a)

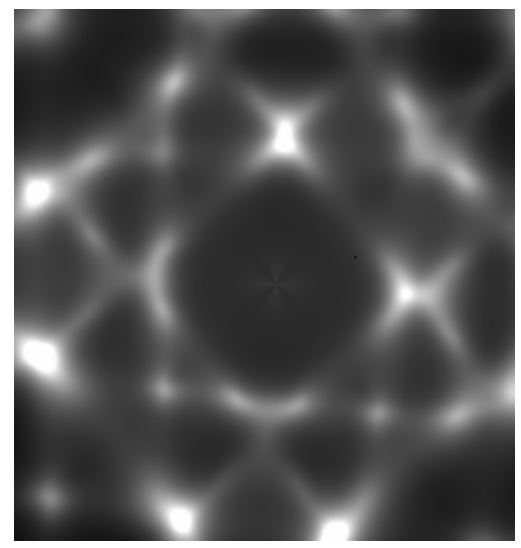

(b)

Fig. 6. (a) Experimental and (b) calculated (fitted) TDS images for $\delta \mathrm{Pu}-\mathrm{Ga}(111),(110)$ and (100) at room . The lead beam stopper appears as a dark circle in each of the experimental TDS patterns in (a)

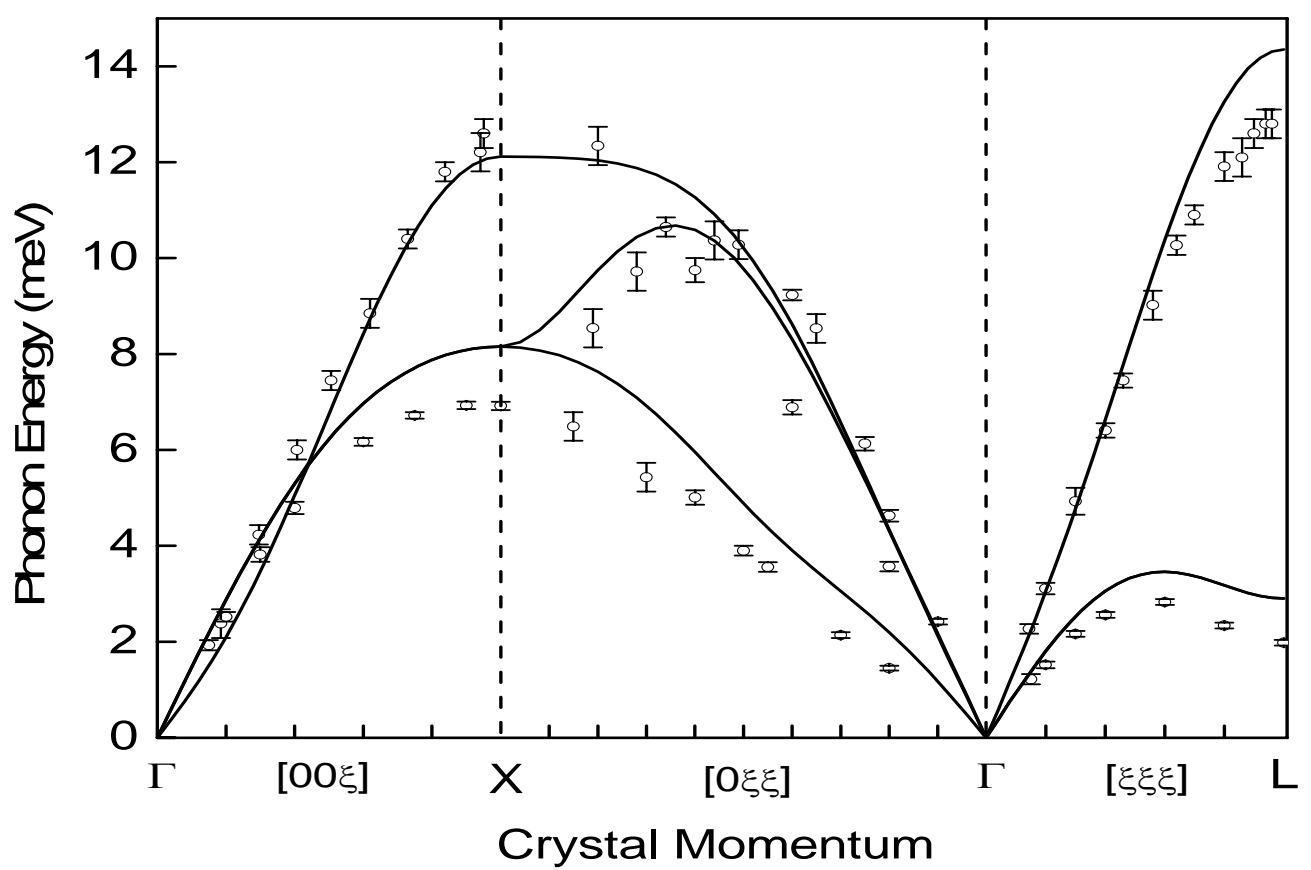

Fig. 7. Calculated phonon dispersions (lines) from fitting of TDS images vs HRIIXS data (points). The solid curves are derived from a best fit to the TDS patterns based on a 4NN Born-von Karman model 
area illuminated by the $\mathrm{x}$-ray beam $(25 \mu \mathrm{m})$ is indeed essentially a single grain.

The images shown in Figs. 6(b) are computed using the method discussed in detail by Holt et al. [Phys Rev. B 66, 064303 (2002)]. Briefly, a Born-von Kármán force constant model ${ }^{18}$ with inter-atomic force constants as input parameters is adopted to calculate the PDCs and the phonon polarization vectors. The x-ray scattering intensities are then computed and fitted to the experimental images shown in Figs. 6(a) simultaneously with the inter-atomic force constants, the grain orientation, background, and overall normalization taken as adjustable fitting parameters. Force constants up to the 4th nearest-neighbor are used. A difficulty here, not encountered in previous TDS studies, is the presence of Debye rings covering a significant portion of the images. These rings could adversely affect the fitting. Fortunately, many of the Debye ring spots have saturated intensities, and these can be easily identified and removed from the fit. For the rest, an algorithm is employed in which a pixel is removed from the fit if its intensity is significantly higher than its neighbors. With this method we could take out most of the extraneous spots. However, the less intense spots and the TDS wings of the intense spots can still contaminate the fitting, resulting in errors in the final answer.

Indeed, fitting in the present case proved to be more problematic than previous TDS studies. Starting from various choices of initial parameters, several local minima in the $\chi^{2}$ value were found. The best fits are shown in Figs. 6(b), and the corresponding phonon dispersion curves are shown in Fig. 7. Also shown in Fig. 7 (circles and error bars) are the experimental data determined by inelastic x-ray scattering $^{8}$. The agreement is clearly not as good as in previous studies of other systems due to the inferior sample quality in the present case. However, it is interesting to note that the present TDS analysis does reveal the phonon softening of the transverse acoustic branch near the L point. This is perhaps the most interesting feature of the Pu PDCs, and its temperature and pressure dependence is an important issue in regard to Pu science and technology. 
Thus, the main purpose of our initial TDS experiments on fcc Pu-Ga alloy was to demonstrate the feasibility of a "large-grain, small-beam" approach in probing phonon properties of difficult materials that are not readily available in the form of large single crystals. Specifically, TDS patterns of acoustic phonons in an fcc $\delta$ Pu-Ga alloy have been successfully recorded for all three major crystallographic orientations. Despite interference of Debye rings arising from inclusions and impurities, the calculated patterns agree reasonably well with experiment, thus validating the general approach of analysis.

\section{Discussion}

\subsection{Born-von Kármán Analysis}

The experimental dispersion curves can be analyzed using the classical Born-von Kármán (B-vK) model with various coordination shells of interacting neighbors. Using a procedure of linear least squares fitting to the dispersions, inter-atomic force constants as well as inter-planar force constants can be determined. ${ }^{20}$ In a fcc lattice there is insufficient information in the dispersion curves along the principal symmetry directions to permit a fit beyond the fourth nearest neighbors (4NN). Constraints on the longrange force constants must be imposed to include higher neighbors. For example, two axially symmetric constraints are needed for a six nearest neighbor $(6 \mathrm{NN})$ model and four for an eight-nearest neighbor $(8 \mathrm{NN})$ model (see Table 2).

A series of B-vK calculations has been performed using models with $2 \mathrm{NN}$ to $8 \mathrm{NN}$ to fit the IXS data. The inter-atomic force constants obtained from these models are listed in Table 2. As seen in Fig. 5 (solid lines), when no constraints are imposed and the forces extending to $4 \mathrm{NN}$ are considered, a reasonable fit to the dispersion curves is obtained. In Fig. 8 , the goodness of fit, $\chi^{2}$, is plotted versus the number of neighbors included in the fit. At $3 \mathrm{NN}, \chi^{2}$ drops significantly but decreases much more slowly upon including additional neighbors. In fact, beyond $4 \mathrm{NN}$, the long-range force constants turn out to be much smaller. Extending the fit to eighth neighbors improves the goodness of the fit somewhat. 


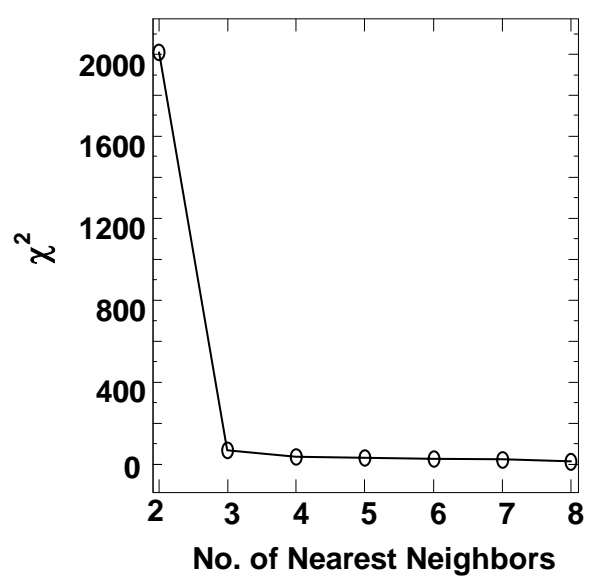

Fig. 8. A statistical estimate of the goodness of fit $\chi^{2}$ for a Born-von Kármán model plotted as a function of the number of nearest neighbors included in the model.

As noted by Dutton et al., ${ }^{21}$ in the fcc lattice, fourth neighbors are the most distant which can be reached in two nearest-neighbor steps. From this structural consideration it seems reasonable that the force constants for neighbors more distant than fourth have distinctly smaller values. Furthermore, it is interesting to note that the $4 \mathrm{NN}$ model for a fcc lattice includes a total of 54 atoms about a central atom: 12 (110)-neighbors from the first coordination shell, 6 (200)-neighbors from the second shell, 24 (211)neighbors from the third and 12 (220)-neighbors from the fourth shell. By composition, the 54 neighbors contain a $\mathrm{Ga}$ atom in the $\mathrm{Pu}-2$ at. \% Ga alloy, implying that the $\mathrm{Ga}$ has to be included in the crystal dynamics of the system within the B-vK framework.

\subsection{Elastic Properties}

The sound velocity, v, associated with each of the phonon branches shown in Fig. 4 can be determined directly from a linear fit to the low q region around the $\Gamma$ point. These velocities are shown in Table 3 and are related to the elastic constants, $C_{i j}$, by the Green-Christoffel equation. ${ }^{22}$ For a cubic crystal, the elastic waves are purely longitudinal or purely transverse along the principal [100], [110] and [111] symmetry directions. The elastic constants for our Pu-Ga alloy given in Table 3 can be computed directly from the following simple relationships ${ }^{22}$ :

$$
\begin{aligned}
& v_{\mathrm{L}}[100]=\left(\mathrm{C}_{11} / \rho\right)^{1 / 2}, v_{\mathrm{T}}[100]=\left(\mathrm{C}_{44} / \rho\right)^{1 / 2}, \\
& v_{\mathrm{L}}[110]=\left(\left[\mathrm{C}_{11}+\mathrm{C}_{12}+2 \mathrm{C}_{44}\right] / \rho\right)^{1 / 2}, v_{\mathrm{T} 1}[110]=\left(\left[\mathrm{C}_{11}-\mathrm{C}_{12}\right] / 2 \rho\right)^{1 / 2}, v_{\mathrm{T} 2}[110]=\left(\mathrm{C}_{44} / \rho\right)^{1 / 2}, \\
& v_{\mathrm{L}}[111]=\left(\left[\mathrm{C}_{11}+2 \mathrm{C}_{12}+4 \mathrm{C}_{44}\right] / 3 \rho\right)^{1 / 2}, v_{\mathrm{T}}[111]=\left(\left[\mathrm{C}_{11}-\mathrm{C}_{12}+\mathrm{C}_{44}\right] / 3 \rho\right)^{1 / 2}
\end{aligned}
$$

where $\rho$ is the mass density. For the $\mathrm{Pu}-2$ at. \% Ga alloy, $\rho$ equals $15.82 \mathrm{gm} / \mathrm{cc}$ at room temperature. 


\section{Table 2. Born von-Karman atomic force constants, $\left(\mathrm{Nm}^{-1}\right)$}

Constraints for $8 N N$ and $7 N N$ : $8(5 Z Z)=9(5 Y Y)-5 X X ; 8(5 X Y)=3(5 X X-5 Y Y) ; 3(7 Y Z)=7 X Y ; 2(7 X Z)=7 X Y$ Constraints for $6 N N$ and $5 N N$ : $8(5 X Y)=3(5 X X-5 Y Y)$ No constraints for $4 \mathrm{NN}, 3 \mathrm{NN}$ and $2 \mathrm{NN}$.

\begin{tabular}{|c|c|c|c|c|c|c|c|}
\hline & $2 \mathrm{NN}$ & $3 N N$ & $4 N N$ & $5 N N$ & $6 N N$ & $7 \mathrm{NN}$ & 8NN \\
\hline $1 X X$ & 8.305 & 8.1149 & 9.4001 & 9.3616 & 9.2694 & 9.145 & 9.0172 \\
\hline $1 Z Z$ & -2.081 & -0.7228 & -2.1735 & -2.2841 & -2.3653 & -2.5584 & -2.1621 \\
\hline $1 X Y$ & 8.557 & 11.3954 & 11.296 & 11.2949 & 11.2981 & 11.4838 & 11.5481 \\
\hline $2 x x$ & -1.929 & -0.7896 & -3.0938 & -2.9988 & -3.0892 & -2.9408 & -2.8591 \\
\hline $2 Y Y$ & -0.707 & -0.1357 & 0.9046 & 0.9719 & 1.1133 & 1.2634 & 0.6954 \\
\hline $3 x x$ & & -1.3769 & -0.5516 & -0.4387 & -0.3527 & -0.4641 & -0.7061 \\
\hline $3 Y Y$ & & 0.4561 & -0.2943 & -0.2915 & -0.2499 & -0.0928 & -0.1324 \\
\hline $3 Y Z$ & & -0.587 & -0.4259 & -0.4245 & -0.4224 & -0.438 & -0.5226 \\
\hline $3 X Z$ & & -0.5389 & -0.3239 & -0.3074 & -0.3014 & -0.3401 & -0.2841 \\
\hline $4 X X$ & & & -0.0657 & -0.0284 & -0.0586 & -0.1854 & -0.0202 \\
\hline $4 Z Z$ & & & 0.9747 & 0.974 & 0.9729 & 1.0115 & 0.7709 \\
\hline $4 X Y$ & & & -0.4969 & -0.538 & -0.5856 & -0.6857 & -0.8681 \\
\hline $5 X X$ & & & & -0.084 & -0.0582 & 0.0141 & 0.3726 \\
\hline $5 Y Y$ & & & & -0.0369 & -0.0141 & -0.1258 & 0.0303 \\
\hline $5 Z Z$ & & & & -0.0015 & -0.0694 & -0.1433 & -0.0125 \\
\hline $5 X Y$ & & & & -0.0176 & -0.0165 & 0.0525 & 0.1284 \\
\hline $6 x x$ & & & & & -0.0698 & -0.1863 & -0.031 \\
\hline $6 Y Z$ & & & & & 0.0255 & 0.0966 & 0.218 \\
\hline $7 X x$ & & & & & & 0.0003 & 0.0425 \\
\hline $7 Y Y$ & & & & & & 0.1319 & -0.0184 \\
\hline $7 Z Z$ & & & & & & -0.048 & -0.0279 \\
\hline $7 Y Z$ & & & & & & -0.0056 & -0.0186 \\
\hline $7 X Z$ & & & & & & -0.0084 & -0.0279 \\
\hline $7 X Y$ & & & & & & -0.0168 & -0.0557 \\
\hline $8 X x$ & & & & & & & -0.9241 \\
\hline $8 Y Y$ & & & & & & & 0.0101 \\
\hline$\chi^{2}$ & 2010.8 & 67.8 & 35.6 & 33.7 & 27.7 & 21.1 & 14.1 \\
\hline
\end{tabular}


Table 3. Sound velocities $v\left(\mathrm{~ms}^{-1}\right)$, and elastic moduli, $\mathrm{C}_{\mathrm{ij}}$ for $\delta \mathrm{Pu}-2$ at. $\%$ Ga (GPa). The elastic moduli for both a Pu-3.4 at. \% Ga alloy by ultrasonics and pure $\delta$ Pu calculated using DMFT are also listed for comparison

$$
\begin{aligned}
& v_{\mathrm{L}}[100]=1586 \pm 20 \quad v_{\mathrm{L}}[110]=1894 \pm 20 \quad v_{\mathrm{L}}[111]=2080 \pm 30 \\
& v_{\mathrm{T}}[100]=1379 \pm 30 \quad v_{\mathrm{T} 1}[110]=566 \pm 4 \quad v_{\mathrm{L}}[111]=979 \pm 45 \\
& v_{\mathrm{T} 2}[110]=1411 \pm 16
\end{aligned}
$$

\begin{tabular}{|c|c|c|c|c|}
\hline System & $\mathrm{C}_{11}$ & $\mathrm{C}_{12}$ & $\mathrm{C}_{44}$ & Remarks \\
\hline$\delta \mathrm{Pu}-2$ at. $\% \mathrm{Ga}$ & $35.3 \pm 1.4$ & $25.5 \pm 1.5$ & $30.53 \pm 1.1$ & This work \\
\hline
\end{tabular}

$\begin{array}{lllll}\delta \mathrm{Pu}-3.4 \text { at. } \% \mathrm{Ga} & 36.28 & 26.73 & 33.59 & \text { Ultrasonics }^{44}\end{array}$

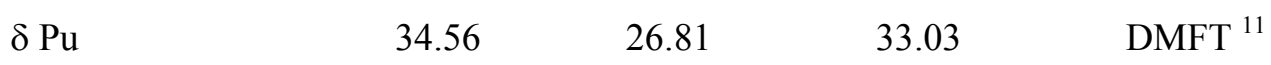

In a cubic crystal, if the interatomic forces are central (ie.: purely radial, angle independent), then the Cauchy relation for the second order elastic constants is fulfilled. ${ }^{23}$

$$
\mathrm{C}_{44}=\mathrm{C}_{12} \text {. }
$$

Deviations from this relation account for the existence of non-central forces. In Table 4, the experimental elastic constants of all fcc metals reported in the literature ${ }^{24-44}$ are listed. Most of these elastic moduli were determined from inelastic neutron scattering, except for those of $\mathrm{Ir}^{25}, \mathrm{Pt}^{26}, \mathrm{Rh}^{27}$ and $\mathrm{Th}^{36}$, which were determined from ultrasonic measurements. The elastic constants for Pt were measured at $90 \mathrm{~K}^{26}$, and those of $\beta-\mathrm{La}^{29}$ and $\gamma-\mathrm{Fe}^{43}$ at $660 \mathrm{~K}$ and $1428 \mathrm{~K}$ respectively at which the fcc phases of these two metals are thermodynamically stable. Deviations from the Cauchy relation, as defined by $\mathrm{C}_{44}-\mathrm{C}_{12}$ are also reported in Table 4. Th appears to be the only fcc metal that obeys the Cauchy equality with $\mathrm{C}_{44} \sim \mathrm{C}_{12}$ (within 0.2 $\%$ ), implying that the interatomic forces in Th are largely central. The rest of the fcc metals exhibit substantial deviation from Cauchy equality, implying that non-central and angular forces are operative in the lattices. Furthermore, the deviation for most fcc metals is negative, ie: $\mathrm{C}_{44}<\mathrm{C}_{12}$, whereas for $\mathrm{Ir}, \gamma$-Ce, $\mathrm{Yb}$, and $\mathrm{SPu}-\mathrm{Ga}$, the deviation is positive, ie. $\mathrm{C}_{44}>\mathrm{C}_{12}$. In the latter three systems, for which the phonon dispersions are known ${ }^{30,42}$, an unusual feature of their dispersion curves is that the $\mathrm{T}$ and $\mathrm{L}$ branches

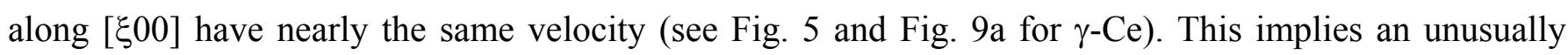
high T-mode sound velocity (and thus a relatively high $\mathrm{C}_{44}$ and a positive $\mathrm{C}_{44}-\mathrm{C}_{12}$ ). In the case of $\delta \mathrm{Pu}$ $\mathrm{Ga}$, it is useful to compare $[\xi \xi 0] \mathrm{T}_{1}$ with $[\xi 00] \mathrm{T}$ (Table 5). One sees comparable first interplanar force

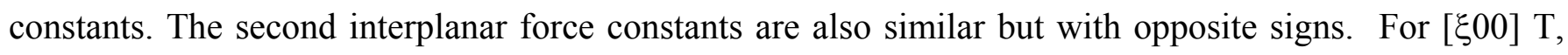
both the first and second terms are positive, resulting in an re-enforcement, making the system more rigid against transverse 
Table 4. Elastic constants $C_{\mathrm{ij}}$ of fec metals (in units of GPa) at $300 \mathrm{~K}$ - Cauchy inequality: $\mathrm{C}_{44}-\mathrm{C}_{12}$, and anisotropy factor: $\mathrm{C}_{44} / \mathbf{1} / 2\left[\mathrm{C}_{11}-\mathrm{C}_{12}\right]$. Except for $\mathrm{Rh}, \mathrm{Ir}$, Pt, Th and Pu-Ga, all elastic moduli were determined from inelastic neutron scattering measurements.

\begin{tabular}{|c|c|c|c|c|c|c|}
\hline Metal & $\mathrm{C}_{11}$ & $\mathrm{C}_{12}$ & $\mathrm{C}_{44}$ & $\begin{array}{c}\text { Cauchy inequality } \\
\mathrm{C}_{44}-\mathrm{C}_{12}\end{array}$ & $\begin{array}{l}\text { Anisotropy factor } \\
2 \mathrm{C}_{44} /\left[\mathrm{C}_{11}-\mathrm{C}_{12}\right\rfloor\end{array}$ & Ref. \\
\hline$\overline{\mathrm{Al}}$ & 106.8 & 60.7 & 28.2 & -32.5 & 1.223 & 24 \\
\hline Ir (ultrasonic) & 600 & 260 & 270 & +10 & 1.588 & 25 \\
\hline $\begin{aligned} \text { Pt } & (90 \mathrm{~K}) \\
& \text { (ultrasonic) }\end{aligned}$ & 346.7 & 250.7 & 76.5 & -174.2 & 1.594 & 26 \\
\hline $\mathrm{Rh}$ (ultrasonic) & 413 & 194 & 184 & -10 & 1.680 & 27 \\
\hline $\mathrm{Ni}$ & 250.8 & 150.0 & 123.5 & -26.5 & 2.450 & 28 \\
\hline$\beta$-La $(300 \mathrm{~K})$ & 34.47 & 20.38 & 17.96 & -2.42 & 2.549 & 29 \\
\hline$\gamma-\mathrm{Ce}$ & 24.1 & 10.2 & 19.4 & +9.2 & 2.79 & 30 \\
\hline $\mathrm{Pd}$ & 227.1 & 176.1 & 71.7 & -104.4 & 2.811 & 31 \\
\hline $\mathrm{Au}$ & 192.3 & 163.1 & 42.0 & -121.1 & 2.876 & 32 \\
\hline $\mathrm{Ag}$ & 124.0 & 93.7 & 46.1 & -47.6 & 3.042 & 33 \\
\hline $\mathrm{Cu}$ & 168.4 & 121.4 & 75.4 & -46.0 & 3.208 & 34 \\
\hline $\mathrm{Ca}$ & 27.8 & 18.2 & 16.3 & -1.9 & 3.396 & 35 \\
\hline Th (ultrasonic) & 75.3 & 48.9 & 47.8 & -0.11 & 3.621 & 36,37 \\
\hline $\mathrm{Sr}$ & 15.3 & 10.3 & 9.9 & -0.4 & 3.960 & 38 \\
\hline $\mathrm{Pb}$ & 49.5 & 42.3 & 14.9 & -27.4 & 4.138 & 39,40 \\
\hline$\beta-\mathrm{La}(660 \mathrm{~K})$ & 28.46 & 20.41 & 16.53 & -3.88 & 4.106 & 41 \\
\hline $\mathrm{Yb}$ & 18.6 & 10.3 & 17.7 & +7.4 & 4.265 & 42 \\
\hline$\gamma-\mathrm{Fe}(1428 \mathrm{~K})$ & 154 & 122 & 77 & -45 & 4.813 & 43 \\
\hline $\begin{array}{l}\delta \mathrm{Pu}-\mathrm{Ga}(\mathrm{IXS}) \\
{[2 \text { at. } \% \mathrm{Ga}]}\end{array}$ & 35.30 & 25.5 & 30.53 & +5.03 & 6.059 & This work \\
\hline $\begin{array}{l}\delta \mathrm{Pu}-\mathrm{Ga} \text { (ultra) } \\
{[3.4 \text { at. \% Ga] }}\end{array}$ & 36.28 & 26.73 & 33.59 & +6.86 & 7.03 & 44 \\
\hline
\end{tabular}


displacement. However, the signs are opposite for $[\xi \xi 0] \mathrm{T}_{1}$. This results in a partial cancellation of the restoring forces, making the lattice softer for the corresponding mode. Thus, [ $\xi 00] \mathrm{T}$ has a larger slope than $[\xi \xi 0] \mathrm{T}_{1}$ resulting in $\mathrm{C}_{44}>\mathrm{C}_{12}$.

Table 4 reports also the anisotropy factor, defined ${ }^{45}$ as the ratio of $\mathrm{C}_{44} / \mathrm{C}^{\prime}$, where $\mathrm{C}^{\prime}=1 / 2\left[\mathrm{C}_{11}-\right.$ $\left.\mathrm{C}_{12}\right]$, associated with the two non-degenerate transverse $\mathrm{T}_{2}[\xi \xi 0]$ and $\mathrm{T}_{1}[\xi \xi 0]$ branches [Equations (1)] in the phonon dispersion curves shown in Fig. 4. In fec metals, the elastic anisotropy factor increases from simple $\mathrm{p}$ metals like $\mathrm{Al}$ to transition metals and rare-earth metals up to a value of $\sim 4$. For the fcc $\mathrm{Pu}-2$ at. $\% \mathrm{Ga}$ alloy, our phonon data shows a high value of 6 . By definition, the large anisotropy factor is directly related to the low speed of sound of $\mathrm{T}_{1}[\xi \xi 0]$, since $2 \mathrm{C}_{44} /\left(\mathrm{C}_{11}-\mathrm{C}_{12}\right)$ is proportional to the ratio of the speed of sound of the two transverse [ $\xi \xi 0]$ phonon branches. This in turn is consistent with the respective values of the interplanar force constants, which are linear combinations of atomic force constants. As seen in Table 5 the first interplanar force constants, $\Phi_{1}$, for the $T_{2}$ and $T_{1}[\xi \xi 0]$ branches are very different, the former being more than 3 times larger than the latter. [This is the main source of the anisotropy.]

\section{Table 5 Interplanar force constants $\left(\mathrm{Nm}^{-1}\right)$ for $\delta \mathrm{Pu}-2$ at. \% Ga calculated from the $4 \mathrm{NN}$ B-vK model}

\begin{tabular}{ccccc} 
Branch & $\Phi_{\mathbf{1}}$ & $\Phi_{\mathbf{2}}$ & $\Phi_{\mathbf{3}}$ & $\Phi_{\mathbf{4}}$ \\
\hline$[00 \xi] \mathrm{L}$ & 70.552 & -11.138 & & \\
{$[00 \xi] \mathrm{T}$} & 22.156 & 3.09 & & \\
& & & & \\
{$[0 \xi \xi] \mathrm{L}$} & 28.136 & 37.802 & -5.98 & -1.128 \\
{$[0 \xi \xi] \mathrm{T}_{2}$} & 72.912 & -3.468 & -2.36 & 1.952 \\
{$[0 \xi \xi] \mathrm{T}_{1}$} & 22.95 & -4.014 & -0.796 & 0.864 \\
& & & & \\
{$[\xi \xi \xi] \mathrm{L}$} & 74.774 & -6.884 & & \\
{$[\xi \xi \xi] \mathrm{T}$} & 1.826 & 2.548 & & \\
\hline
\end{tabular}

The present result confirms an earlier ultrasonic measurement on a higher Ga (3.4 at. \%) alloy by Ledbetter and Moment ${ }^{44}$ who reported an anisotropy factor of 7 and discussed the elastic properties of $\delta \mathrm{Pu}-\mathrm{Ga}$ alloys in detail. The extremely high elastic anisotropy with regard to propagation of elastic waves in $\mathrm{fcc} \mathrm{Pu}$ is also substantiated by a recent dynamical mean field theory (DMFT) calculation ${ }^{11}$ which yielded an even higher theoretical anisotropy factor of 8 for pure $\delta$-Pu. More recent DFT 
(density functional theory) calculations ${ }^{46}$ using various formalisms to describe several assumed magnetic configurations of $\delta$-Pu (disordered, ferromagnetic, non-magnetic) yields anisotropy factor values ranging from 1.6 to 10 , and not a single set of elastic constants from a given formalism agrees with those experimentally determined in this study to within a factor of two.

\subsection{Phonon Density of States, Lattice Heat Capacity and Debye Temperature}

Using the phonon dispersion relations derived from the 4NN Born-von Kármán model shown in Fig. 5, the phonon density of states (DOS) has been computed. The computation was carried out using a grid of $(2 \pi / a) / 500$, and the result is normalized such that the area under the curve corresponds to 3 states per atom. A Gaussian smoothing by $0.03 \mathrm{meV}$ was applied to produce the final plot shown in Fig. 9(a). The peak at $11.7 \mathrm{meV}$ in the DOS contains major contributions from all three $\mathrm{L}$ branches and the $\mathrm{T}_{2}(011)$ branch near the $\mathrm{X}$ point. The peak at $6.2 \mathrm{meV}$ has contributions from all branches except the T(111) branch which contributes most significantly to the "rounded-off" feature at $2.1 \mathrm{meV}$. Critical points obtained from the symmetry points in the zone are indicated in the DOS plot.

The DOS has been used to compute the lattice specific heat, $\mathrm{C}_{\mathrm{v}}$, as a function of temperature, and the result is presented in Fig 9(b). At high temperature, $\mathrm{C}_{\mathrm{v}}$ approaches the classical Dulong and Petit limit of $3 R=5.96$ cal.mole $\mathrm{K}^{-1}$, where $R$ is the molar gas constant. At low temperature, the electronic contribution, $\gamma T$, to the total heat capacity becomes significant. ${ }^{47,48}$ Indeed, by measuring the total heat capacities of a $\delta-\mathrm{Pu}_{0.95} \mathrm{Al}_{0.05}$ alloy and pure $\alpha-\mathrm{Pu}$ at low temperatures down to $2 \mathrm{~K}$ and

subtracting the lattice contribution derived from inelastic neutron scattering data ${ }^{49}$, Lashley et al. ${ }^{50}$ found that the $\gamma$ value for the $\delta \mathrm{Pu}-\mathrm{Al}$ alloy is almost a factor of 4 higher than that for pure $\alpha-\mathrm{Pu}$, suggesting proximity of the $\delta$ phase to a quantum critical point.

From the computed lattice specific heat, the Debye temperature, $\Theta_{\mathrm{D}}$, has also been computed as a function of temperature. This is plotted in Fig. 9(c). Above 100K, the Debye temperature, $\Theta_{\mathrm{D}(\infty)}$, remains remarkably constant at a value of $\sim 119 \mathrm{~K}$. At $0 \mathrm{~K}$, the calculated value $\Theta_{\mathrm{D}(0)}$ is $114 \mathrm{~K}$, which is in excellent agreement with the values of $115 \mathrm{~K}$ and $120 \mathrm{~K}$ determined respectively from ultrasonic 

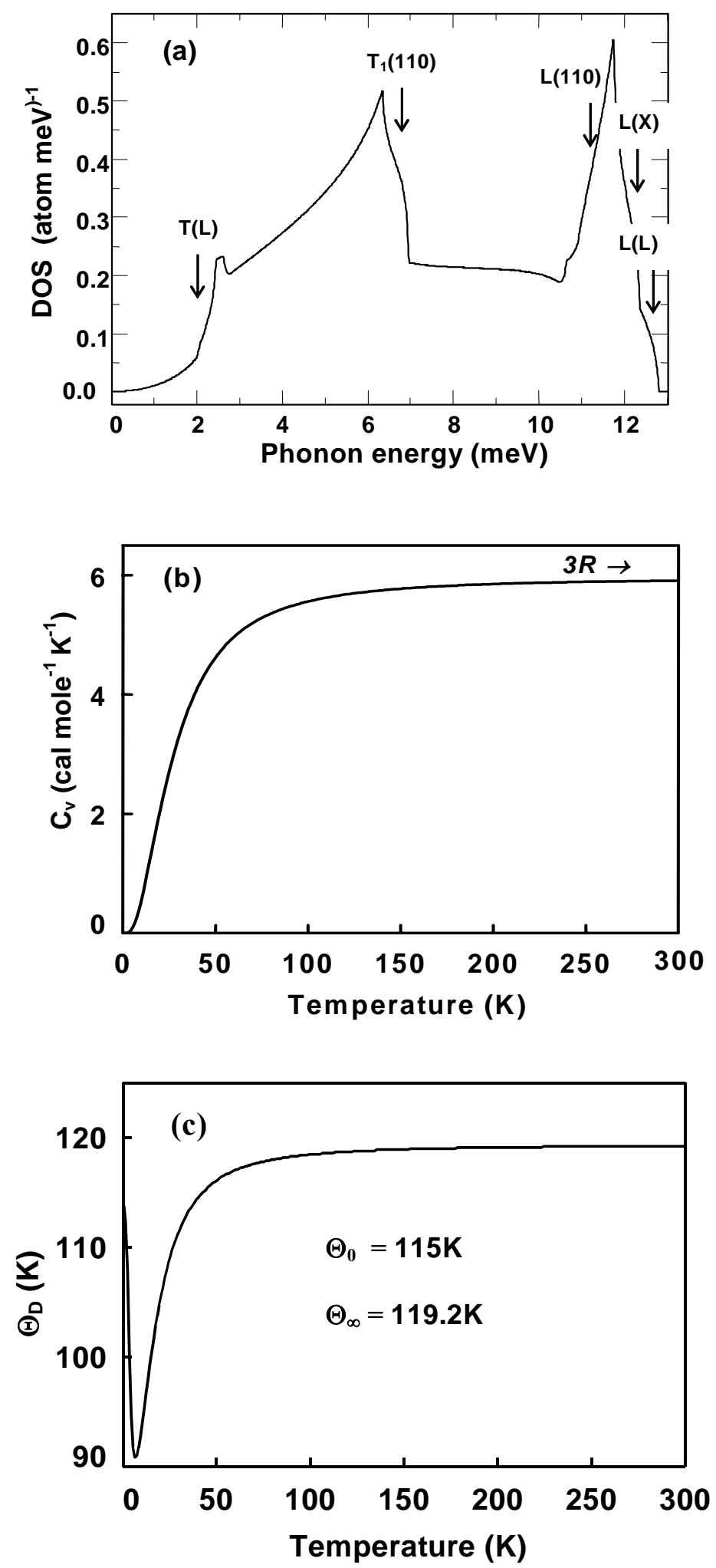

Fig. 9. (a) The density of states (DOS), (b) lattice specific heat and (c) Debye temperature of an fcc Pu-2 at. \% Ga alloy calculated from a 4NN Born-von-Kármán model. Critical points in the DOS obtained from the symmetry points in the zone are indicated in (a). 
measurements ${ }^{44}$ on a $\delta$ Pu-3.4 at. \% Ga alloy and from EXAFS measurements ${ }^{51}$ on a $\delta$ Pu-1.9 at. \% Ga alloy. In Table 6 the Debye temperatures of fcc metals calculated from phonon DOS derived from Born-von Kármán modeling of experimentally determined phonon dispersions are tabulated. It is interesting to note that $\mathrm{Au}, \gamma-\mathrm{Fe}$, and $\delta \mathrm{Pu}-\mathrm{Ga}$ are the only fcc metals with $\Theta_{\mathrm{D}(0)}<\Theta_{\mathrm{D}(\infty)}$. At low temperatures, only the low frequency modes are thermally occupied. A low Debye temperature implies a low average speed of sound. Thus, the average speed of sound in $\mathrm{Pu}-\mathrm{Ga}$ is lower than what one might expect based on the high-temperature limit of the Debye temperature. The "positive dispersion" in the $\mathrm{T}_{1}[\xi \xi 0]$ branch (discussed later in Section 5) might be responsible for the low Debye temperature at $\mathrm{T}=0$. Since at low temperatures, only the phonons around the $\Gamma$ point contribute, a lower Debye temperature means a lower average speed of sound near the $\Gamma$ point, which fits the behavior of the $\mathrm{T}_{1}[[\xi \xi 0]$ branch well. This branch has the lowest speed of sound (i.e., the lowest phonon energy near Г) hence, the largest phonon population, and thus may be the major cause of the unusual trend in the Debye temperature. Similarly, $\mathrm{Au}^{32}$ and $\gamma-\mathrm{Fe}^{43}$ have a positive dispersion in the $\mathrm{T}_{1}[\xi \xi 0]$ branch and both metals also have lower Debye temperatures at $\mathrm{T}=0$.

It should be noted that the above discussion on Debye temperature is based on the dispersion curves measured at room temperature. As the temperature changes, the dispersion curves will likely change, and in fact, at $\sim 170 \mathrm{~K}$ the fcc Pu-Ga alloy undergoes a phase transition to a monoclinic $\alpha$ phase $^{5}$ (see Section 4 below). Thus, the Debye temperature at $\mathrm{T}=0$ as seen in Fig. 9(c) is not the same Debye temperature measured at $\mathrm{T}=0$.

\section{4 $T(\xi \xi \xi)$ Branch Bending and the $\delta \rightarrow \alpha$ 'Transformation}

The experimental PDCs shown in Fig. 5 display a pronounced bending towards lower energies of the $\mathrm{T}[\xi \xi \xi]$ branch near the L point. A similar feature (but occurring at about twice the energy and at a higher crystal momentum towards the L point) is also seen in a recent dynamic mean field theory (DMFT) calculation of the PDCs of $\delta$-Pu ${ }^{11}$, (Fig.10). The bending of this $\mathrm{T}[\xi \xi \xi]$ mode is rather unusual in fcc metals, occurring in only two other systems: $\gamma-\mathrm{Ce}^{30}$ and $\beta$ - $\mathrm{La}^{29,}{ }^{41}$, whose PDCs determined by inelastic neutron scattering are reproduced in Fig. 11. Temperature dependent studies have shown that the $\mathrm{T}[\xi \xi \xi]$ modes in these two rare-earth systems soften near the $\mathrm{L}$ point at low temperature. Based on this evidence, it has been proposed that the observed phonon softening is 


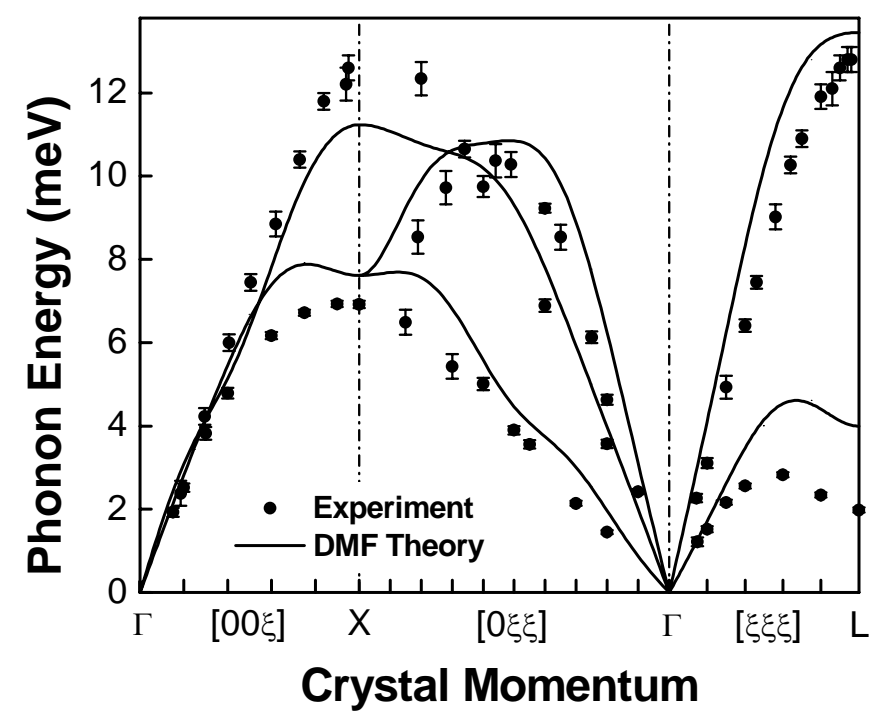

Fig. 10. Comparison of calculated phonon dispersions (lines) for pure $\delta \mathrm{Pu}$ using DMFT-linear response theory by Dai et al. ${ }^{11}$ with the present experimental dispersions (circles) for an fcc $\delta \mathrm{Pu}-\mathrm{Ga}$ alloy, showing overall qualitative agreement.

related to the $\mathrm{fcc} \rightarrow$ dhcp phase transformation which occurs in $\mathrm{Ce}$ and $\mathrm{La}$ at $283 \mathrm{~K}$ and $660 \mathrm{~K}$ respectively. ${ }^{30}$ By analogy, the observed bending of the $\mathrm{T}[\xi \xi \xi]$ branch in the present experiment on the Pu-Ga alloy may be related to the $\delta \rightarrow \alpha$ 'transformation which occurs upon cooling these materials to sub-ambient temperatures. ${ }^{5,52}$ Measurements of the temperature dependence of the PDCs are essential to validate this hypothesis. The softening of the $\mathrm{T}[\xi \xi \xi]$ branch is also consistent with the crystallography of transformations that occur in $\gamma$-Ce, $\beta$-La, and $\delta \mathrm{Pu}-\mathrm{Ga}$ alloys. In all cases, the parent phase is the fcc crystal structure which is composed of hexagonal-close-packed atomic planes stacked along the [111] direction with an $A B C A B C \ldots$ stacking arrangement. The soft transverse mode at $\mathrm{L}$ suggests that a (111) plane could be easily sheared relative to its neighboring atomic planes to form new stacking arrangements. In the case of $\mathrm{Ce}$ and $\mathrm{La}$ the product phase has a dhcp structure with an $A B A C A B A C \ldots$ stacking arrangement, while in the case of $\mathrm{Pu}-\mathrm{Ga}$ alloys the product phase is a complex monoclinic structure ${ }^{53}$ which can also be viewed as a distorted hexagonal-close-packed structure with an $A B A B A B \ldots$ stacking arrangement. It is also possible that the phonon softening is related to the $\delta \rightarrow \gamma$ transformation which occurs in pure $\mathrm{Pu}$ and produces a phase with a face-centered orthorhombic structure $^{54}$ which can also be viewed as a stack of slightly distorted hexagonal packed atomic layers 


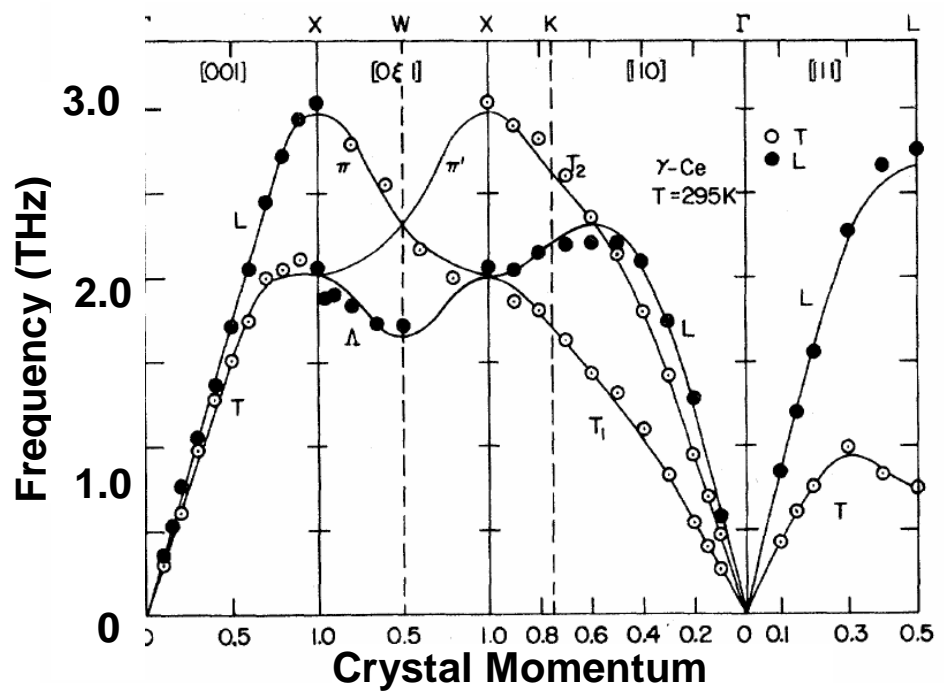

(a)

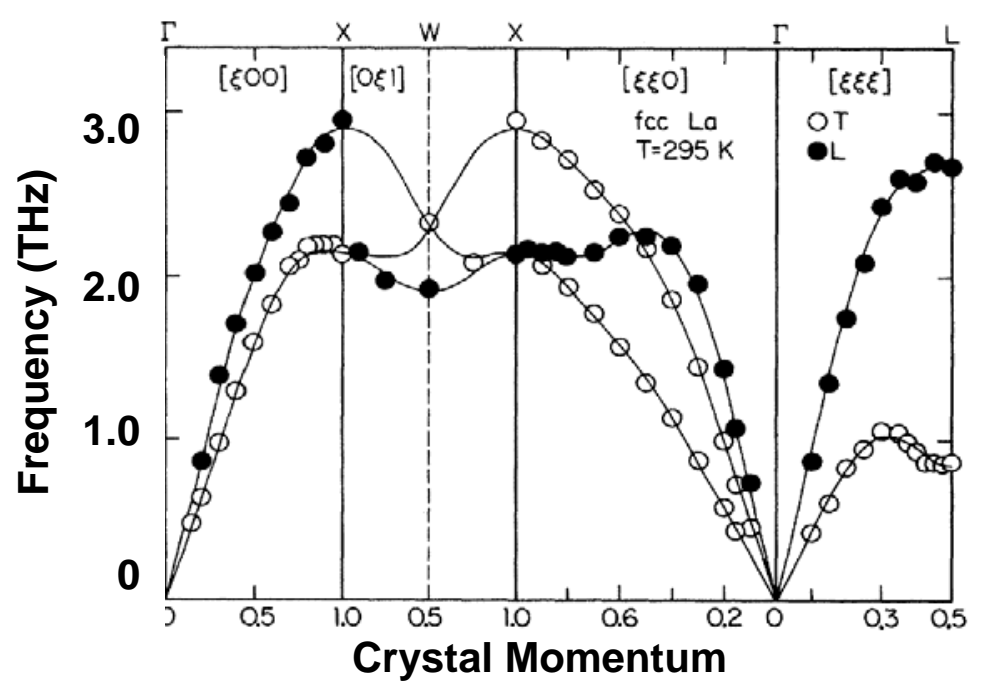

(b)

Fig. 11. Room temperature phonon dispersion curves of (a) $\mathrm{fcc} \gamma-\mathrm{Ce}^{30}$ and (b) fcc $\beta-\mathrm{La}^{29}$, showing bending of the $\mathrm{T}[\xi \xi \xi]$ branch near the $\mathrm{L}$ point similar to that observed in the current $\mathrm{Pu}-\mathrm{Ga}$ alloy. In each case, the smooth curves represent a $8 \mathrm{NN}$ Born-von Kármán force-constant model fit to the inelastic neutron scattering data. (Reproduced with permission from the authors)

which have an $A B A B A B \ldots$ stacking arrangement. The low energy of the necessary shearing processes involved in these transformations can be seen in the first interplanar force constant of the $\mathrm{T}[\xi \xi \xi]$ mode (as derived from the 4NN B-vK model) which is more than an order of magnitude smaller than those of the transverse modes in the other two directions, (see Table 5). 
In order to more precisely relate the bending of the T[ $\xi \xi \xi]$ branch to the $\delta \rightarrow \alpha^{\prime}$ transformation in Pu-Ga alloys, we can employ the crystallographic models that have been developed by Adler and Olsen $^{55}$, and more recently by Jin et al. ${ }^{56}$ These models use as a starting point the crystallographic correspondence $(111)_{\delta} \|(020)_{\alpha}$ and $[-110]_{\delta} \|[100]_{\alpha}$ which has been established using transmission electron microscopy by Zocco et al. ${ }^{57}$ Both sets of models predict stable transformation twins with $(205)_{\alpha}$ twin planes also in agreement with the TEM observations. The distortion required to transform a region of the fcc $\delta$ phase crystal structure to the monoclinic $\alpha^{\prime}$ phase crystal structure can be decomposed into three elementary distortions.

(i). A shuffling operation transforms the fcc structure into an hep structure by changing the stacking sequence of $\{111\}$ planes

(ii). A homogeneous elastic strain distorts the hep structure such that an enlarged 16 atom unit cell has lattice vectors which match the lattice vectors of the $\alpha$ ' phase.

(iii). A set of periodic displacements modify the basis vectors of the distorted, enlarged hep unit cell such that the $\alpha^{\prime}$ crystal structure is formed. In this step the lattice vectors do not change, only the internal coordinates of the atoms with respect to these vectors. Thus this step does not produce any elastic strain.

The observed low phonon energy in $\delta \mathrm{Pu}-\mathrm{Ga}$ near the $\mathrm{L}$ point can be related to the shuffling operation described above. The shuffling operation requires that alternating (111) planes in the fcc lattice be rigidly translated in order to produce the hcp lattice. There are an infinite number of ways in which a shuffling can transform the fcc lattice into the hcp lattice. The simplest mode is a single elementary shuffle and is used in the correspondence suggested Adler and Olsen ${ }^{55}$ which agrees with the TEM observations. ${ }^{57}$ In this case, every other (111) layer is rigidly shifted by $1 / 6[-211]$. This shuffling operation is a combination of a zone boundary $\mathrm{T}[\xi \xi \xi]$ phonon and a homogeneous shear parallel to the (111) plane. Thus, the experimentally observed bending of the $\mathrm{T}[\xi \xi \xi]$ branch is consistent with the proposed model, but we should emphasize that it is only one component of the shuffle operation which itself is only one part of the entire transformation process.

Other more complex shuffling modes are also considered by Jin et al. ${ }^{56}$ They all involve rigidly shifting $\{111\}$ planes to modify the stacking sequence. In these cases, the shuffle includes one or more transverse phonons propagating in the $<111>$ direction but with different wave-vectors. Again, a 
homogeneous shear parallel to the $\{111\}$ plane is necessary to complete the shuffle in addition to the appropriate phonon. Each of these proposed shuffle modes lead to different predictions for the habit plane and volume fraction of each twin variant. Further experimental data is needed to clarify which shuffle mode is involved in the phase transformation. In all cases however, the low energy of the $\mathrm{T}[\xi \xi \xi]$ branch near the $\mathrm{L}$ point is consistent with the crystallographic models of the $\delta \rightarrow \alpha$ ' transformation.

\subsection{Anomaly in the $T_{1}(\xi \xi 0)$ Branch}

In Fig. 5 the $\mathrm{T}_{1}$ branch along [ $\left.\xi \xi 0\right]$ exhibits a "kink" with positive dispersion towards the $\mathrm{X}$ point, similar to those observed in other fcc metals such as $\mathrm{Th}^{36}, \mathrm{Au}^{32}, \mathrm{Pt}^{26}$ and $\mathrm{Pd}^{31}$ The existence of the kink in the $\mathrm{T}_{1}[\xi \xi 0]$ dispersion can graphically be shown from the dispersion data shown in Fig. 5. If one draws a linear dotted line from the $\Gamma$ point through the one or two nearest data points to make a $\mathrm{n}$ extension of the linear range of the dispersion curve, then the rest of the data points along the branch should usually distribute below this line for a normal branch (dash lines). However, this is not the case for the $\mathrm{T}_{1}[\xi \xi 0]$ branch (dotted line), which shows clearly that the slope undergoes a positive upturn (kink) as we move away from the $\Gamma$ point. Moreover, the fitting results from the $4 \mathrm{NN}$ (solid lines in Fig. 4) to $8 \mathrm{NN}$ B-vK models all show an "upturn" in the $\mathrm{T}_{1}[\xi \xi 0]$ branch. This kink also appears in the DMFT results of Dai et al. ${ }^{11}$ shown in Fig. 7.

By analogy with other phonon anomalies observed in metals, the observed upturn in the $\mathrm{T}_{1}[\xi \xi 0]$ branch is likely to be caused by electronic effects. ${ }^{19}$ As the speed of sound of this mode is given by $\sqrt{C^{\prime} / \rho},(\rho=$ density $)$, it is clear that this anomaly is directly responsible for the small value of $\mathrm{C}^{\prime}$ and implies a soft response of the system to a volume-conserving tetragonal distortion. This anomaly has been associated ${ }^{8}$ with the phase transformations of $\mathrm{Pu}$ at high temperature from $\delta \rightarrow \delta^{\prime}$ (body centered tetragonal) $\rightarrow \varepsilon$ (body centered cubic). These transformations could involve just a tetragonal distortion of this type via the so-called Bain path. ${ }^{58}$

\subsection{Theoretical Considerations}

As evident in Fig. 10, the present $\mathrm{x}$-ray results validate the main qualitative predictions of a recent DMFT dispersion calculation by Dai et al. for $\delta$-Pu ${ }^{11}$ in terms of, (i) a large bending towards 
lower energy of the $\mathrm{T}[\xi \xi \xi]$ branch near $\mathrm{L}$, (ii) a kink in the $\mathrm{T}_{1}[0 \xi \xi]$ branch, and (iii) a low shear elastic modulus C'. Such experimental-theoretical agreements lend credence to the DMFT approach for theoretical treatments of correlated $5 f$ electron systems as exemplified by $\delta$-Pu. Quantitatively however, differences exist. These are: position of the energy maximum along the $\mathrm{T}[\xi \xi \xi]$ branch, position of the Kohn-like kink along the $\mathrm{T}_{1}[0 \xi \xi]$ branch, and bending of the calculated $\mathrm{T}[\xi 00]$ branch near the $\mathrm{X}$ point, which is not observed experimentally in the fcc Pu-Ga alloy.

Dai et al. ${ }^{11}$ also calculated the phonon dispersion curves for the bcc $\varepsilon$-Pu phase with the DMFT method and found that several modes are totally unstable at $\mathrm{T}=0$. Existence (stability) of $\varepsilon$-Pu at high temperature $^{3}$ was attributed to anharmonicity and final temperature phonon entropy ${ }^{11}$. On the other hand, the calculated phonon dispersion curves for $\delta-\mathrm{Pu}$ shown in Fig. 6 were also computed at $\mathrm{T}=0$. All modes are found to be stable (no negative frequencies) at all $\boldsymbol{q}$-values, in spite of the fact that pure $\delta$-Pu is the equilibrium phase only at high temperature in the range $592-724 \mathrm{~K}^{3}$

More recently, using a simple inclusion of electron correlations in a Friedel Model of the density of $f$-electron states, and an empty-core pseudopotential treatment for the three free electrons per atom, Harrison ${ }^{59}$ calculated the phonon dispersion curves of $\delta$-Pu, which contain neither the kink in the $\mathrm{T}_{1}[\xi \xi 0]$ branch nor any bending in the $\mathrm{T}(\xi \xi \xi)$ branch observed experimentally in this study. The derived elastic constants were also in poor agreement with both the earlier ultrasonic work ${ }^{44}$ and our IXS data. The predicted anisotropy factor was 19 compared with an experimental factor of 6-7 (Table 4). These differences between the experimental and calculated phonon dispersions are significant and thus provide the framework for refined theoretical treatments ${ }^{12}$ and further experiments in Pu and other $5 f$ systems.

\section{Other Accomplishments}

\subsection{Publications}

Crystal dynamics of $\delta f c c P u$-Ga by high resolution inelastic $x$-ray scattering, Joe Wong, M.Krisch, D. Farber, F. Occelli, R. Xu, T-C. Chiang, D. Clatterbuck, A. Schwartz, M. Wall, and C. Boro, Phys. Rev. B 72, 064115 (2005)

Probing phonons in plutonium,

Joe Wong, M. Krisch, D. Farber, F. Occelli, A. Schwartz, T.C. Chiang, M. Wall, C. Boro 
and Ruqing Xu， Mat. Res. Soc. Symp. Proc. Vol. 802, DD2.2.1 (2004)

Imaging of phonons in an $f c c P u$-Ga alloy by thermal diffuse $x$-ray scattering,

Joe Wong, M. Wall, A. Schwartz, R. Xu, M. Holt, H. Hong, P. Zschack and T-C.Chiang, Appl. Phys. Lett, 84, 3747 (2004)

Phonon Dispersion of fcc $\delta P u-G a$ by Inelastic X-ray Scattering,

Joe Wong, M. Krisch, D. Farber, F. Occelli, A. Schwartz, T-C. Chiang, M. Wall, C. Boro and R. Xu, Science, 301, 1078 (2003)

Our Pu phonon papers have generated a considerable amount of scientific and PR interest, and resulted in a number invited talks, awards, and writeups, both internal and external:

\subsection{Invited talks}

Physics Seminar, Advanced Photon Source, Argonne National Laboratory, Argonne, IL

"Catching Phonons in Plutoniium"

$(\mathrm{Oct} / 03)$

Materials Physics Seminar, Federick Seitz Materials Research Lab. Univ. of Illinois, Urbana IL

"Catching Phonons in Plutoniium"

$(\mathrm{Oct} / 03)$

JOWOG32 Materials Research Meeting, Los Alamos National Laboratory, Los Alamos, NW

"Catching Phonons in Plutoniium"

2003 MRS Fall Meeting, Symposium DD on Actinide Science and Technology, Boston MA

"Phonon Dispersions in fcc $\delta$ Pu-Ga alloy by IXS"

$(\mathrm{Dec} / 03)$

ALS X-ray Science and Technology Seminar, Berkeley CA

"Probing Phonons in Plutonium using High resolution Inelastic x-ray scattering"

(Mar/04)

Amer. Phys. Soc. March Meeting: Symposium on Novel Pu-containing Materials, Montreal, Canada

"Full Phonon Dispersions in fcc Pu-Ga by High Resolution Inelastic X-ray Scattering" (Mar/04)

Gordon Conference on High Pressure, Meriden, NH,

"Probing Phonons in Plutonium using HRIXS"

(Jun/04)

$3^{\text {rd }}$ International Conference Workshop on Electron Correlations \& Materials Properties, Kos Greece "Lattice dynamics in $\delta$ fcc Pu-Ga by Inelastic X-ray Scattering"

Phonons 2004 - International Conference on Phonons Physic, St. Petersburg, Russia

"Phonon dispersion curves in $\delta$ fcc Pu-Ga by Inelastic X-ray Scattering"

$(\mathrm{Jul} / 04)$

Joint Physics Colloquium, Universities of Dortmund and Wuppertal, Dortmund, Germany

"Phonons and Lattice dynamics in $\delta$ fcc Pu-Ga using Inelastic X-ray Scattering"

(Aug/04)

$3^{\text {rd }}$ Workshop on Actinide-XAS 2004, Advanced Light Source, Berkeley, CA

"Phonon dispersions in $\delta$ fcc Pu-Ga by Inelastic X-ray Scattering"

$(\mathrm{Sept} / 04)$

Actinide Workshop at the ALS User Meeting, Berkeley, CA

"Crystal dynamics in $\delta$ fcc Pu-Ga using High Resolution Inelastic X-ray Scattering"

$(\mathrm{Oct} / 04)$ 
Advancing Materials R\&D for National Security at DOE User Facilities, LANL, Los Alamos

"Phonon dispersions and phase stability in fcc $\delta$ Pu-Ga alloys using HRIXS"

XX International Union of Crystallography Congress, Florence, Italy,

"Lattice dynamics and phase transformation in fcc $\delta \mathrm{Pu}-\mathrm{Ga}$ alloys using HRIXS"

$($ Aug/05)

Internal presentation:

CMS DRC Review

B-Division Work in Progress $\quad$ (7/03)

NNSA LDRD Review $\quad(9 / 03)$

DNT DRC Review $\quad(12 / 03)$

Campaign-2 Review $\quad(1 / 04)$

\subsection{Awards:}

- 2005 Fellow of the American Physical Society to JW

- 2003 Director Award for Science and Technology

- 2003 CMS Associate Director Publication Award

- 2003 MSTD Division Publication Award

\subsection{PR Writeups}

- Science Editorial in August 22 issue, 2003

- Science Perspective by Prof. Dr. G. Lander, (Aug, 2003)

- LLNL Newsline (Aug 22, 2003)

- TriValley Herald (Aug. 23 2003)

- La Recherche (France, Nov, 2003)

- Actinide Review Quarterly (LANL, Nov 2003)

- Phyzik Journal (Germany, Nov 2003)

- LLNL S\&TR (Jan-Feb, 2004)

- LLNL 2003 LDRD Highlights (March, 2004)

- ESRF 2003 Highlights (Feb, 2004)

- CMS Strategic Plan, UCRL-TR-203305 (April, 2004)

- CMS 2003 Annual Report, (June, 2004)

- LLNL 2003 Annual Report, (June, 2004)

- LLNL 2005 Newsline (Dec, 9 \& 16)

\subsection{Text Books}

Our Pu-Ga phonon dispersion curves are already appearing in two recent text books:

- "X-ray Optics: High Energy Resolution Applications" by Y. Shvyd'ko, Springer-Verlag (2005).

- Transport in Multilayer Nanostructures: The DMFT approach, by J. Frederick, Imperial College Publishers, London, 2006 (in press) 


\section{Impact, Future Work and Concluding Remarks}

\subsection{Impact}

Today, the actinides are at the frontier of both electronic-structure theories and lattice dynamics research. This research focuses directly on a very strategic LLNL investment area common to both DNT and CMS - the area in actinide science critical to our actinide science and technology. It also addresses issues of concern to DNT's campaigns regarding material dynamics, stability, and aging. The ultimate product of this unique investigation is the acquisition of the much-needed phonon dispersions (including the single crystal elastic moduli) in $\delta \mathrm{Pu}-\mathrm{Ga}$ alloys, and their composition, and temperature dependencies that govern the phase stability, equation of state and a host of critical physico-chemical properties of Pu-Ga alloys. The bona fide phonon data will be used to advantage for both understanding and predicting the phase stability, and aging properties of $\mathrm{Pu}-\mathrm{Ga}$ alloys. Furthermore, our (first) measurements of phonons in plutonium provide a unique and crucial constraint on the interatomic potentials that underlie computer simulations of equations of state that is critical to the science-based stockpile stewardship program.

\subsection{Future Work}

As we can see, the bending of the TA(111) branch towards the L point in $\delta \mathrm{Pu}-\mathrm{Ga}$ is perhaps the most scientifically intriguing in light of the crystallographic correspondence for the $\delta \rightarrow \alpha$ ' transformation discussed in Section 4.4. We have designed, fabricated and test successfully on line at ESRF a cryo-chamber with a micro-jet system (Fig. 12) for IXS measurements down to 200K with 0.1 $\mathrm{K}$ stability over $30 \mathrm{~h}$ and more. We had attempted IXS measurements at ambient and low temperatures, and reproduced our earlier room temperature bending of the TA(111) branch (Fig. 13). At low temperatures however, we measured only the quasi elastic peak, but not the phonon peaks. This might arise from various stresses induced in the sample due to the mounting configuration and other factors. Future work has to be directed towards these mounting as well as sample quality issues.

\subsection{Concluding Marks}

In this project we have rendered a crystal dynamics analysis of the phonon dispersion curves (PDCs) of an fcc $\delta \mathrm{Pu}-\mathrm{Ga}$ alloy determined recently ${ }^{8}$ using a microbeam on large grain sample 

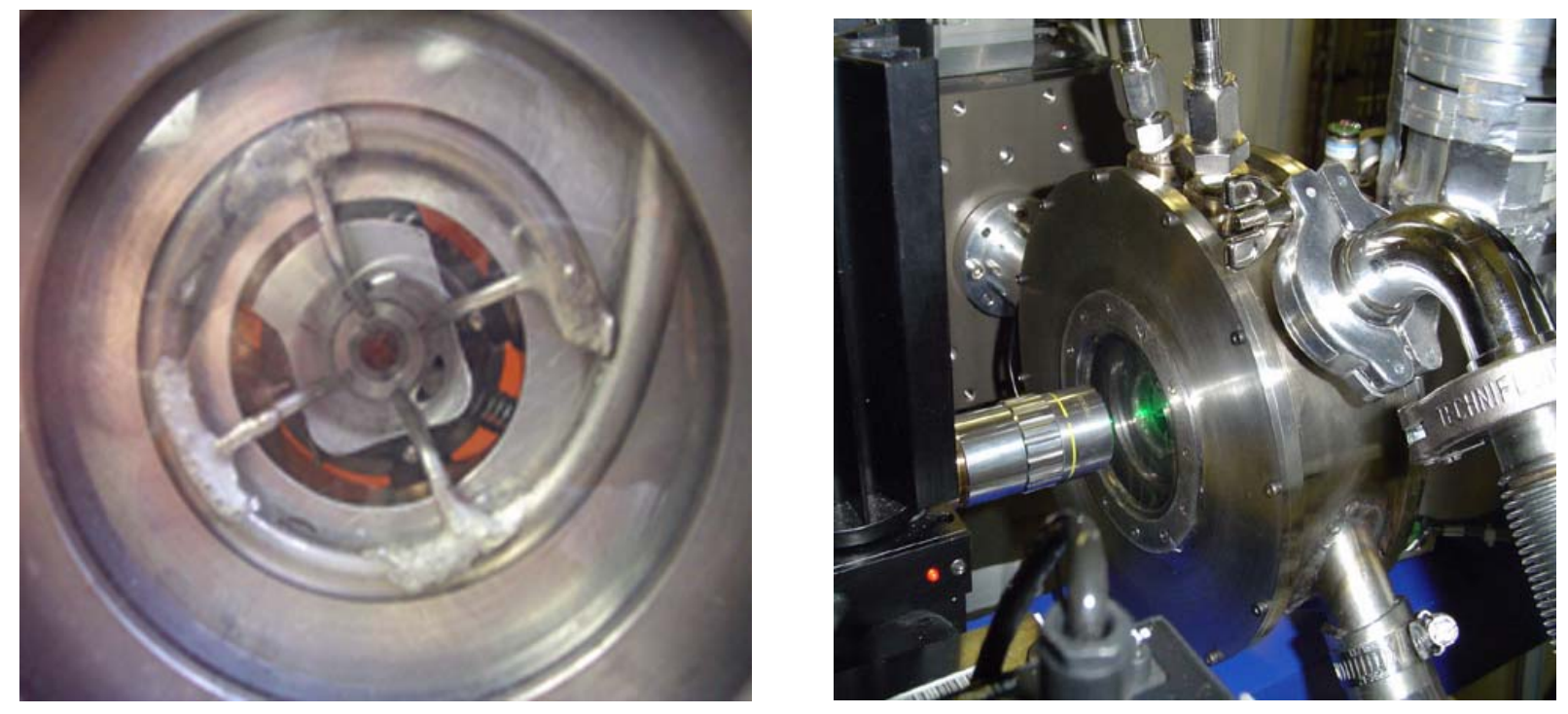

Fig. 12. Micro-jet system (left) for cooling Pu-Ga specimens for HRIXS measurements (right) at ESRF.

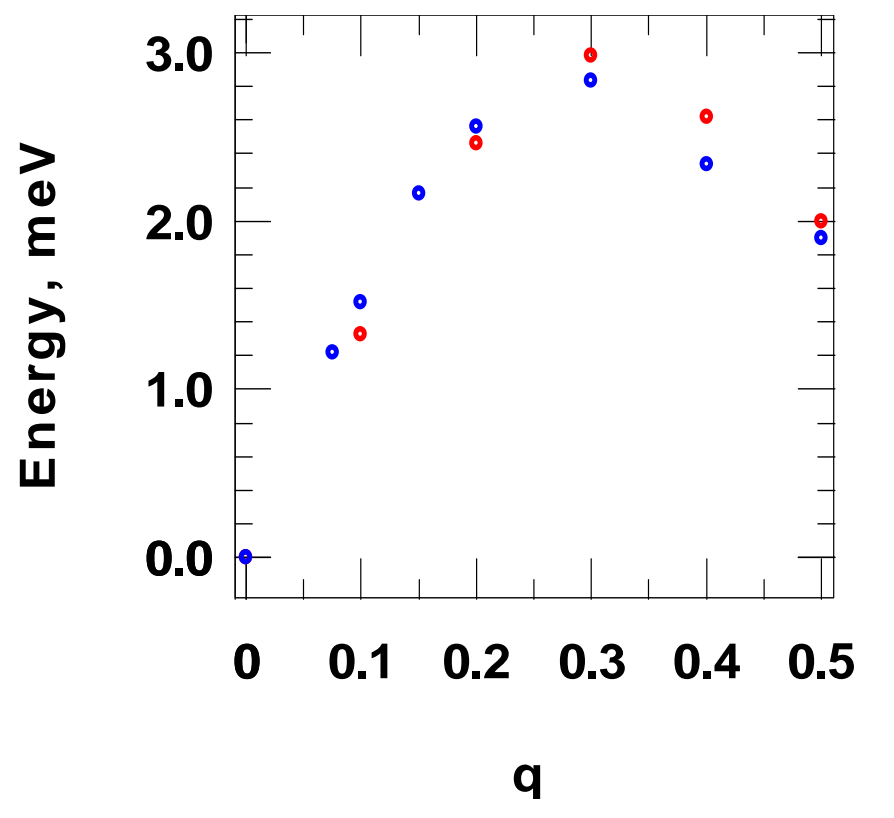

Fig. 13. The bending of the T(111) branch is reproducible: 2003 data (blue) and 2005 data (red).

experimental concept with high resolution inelastic $\mathrm{x}$-ray scattering to obviate the roadblocks of phonon dispersion measurements on Pu-bearing materials with conventional inelastic neutron scattering. Our PDC data confirms directly the very high elastic anisotropy of fcc $\mathrm{Pu}-\mathrm{Ga}$ alloys (highest of all known fcc metals), first discovered almost 3 decades ago from very careful ultrasonic measurements ${ }^{44}$. More important is the pronounced bending observed for the $\mathrm{T}[\xi \xi \xi]$ branch near the $\mathrm{L}$ 
point. The low energy of this branch is found to be crystallographically consistent with the metastability of the fcc phase of the Pu-Ga alloy and its transformation to a pseudo hexagonal-closed-pack, monoclinic $\alpha$ ' phase via a martensitic transformation. A temperature dependence study of the $\mathrm{T}[\xi \xi \xi]$ branch, which is now underway, will hopefully clarify the role of lattice dynamics in the $\delta \rightarrow \alpha$ ' transformation in Pu-Ga alloys. This study provides a needed bona-fide data set for realistic calculations and simulations of thermodynamic and other physical properties for $\mathrm{Pu}$ and its alloys. The phonon dispersion results also provide a critical test for theoretical treatments of highly correlated $5 f$ electron systems, as exemplified by recent dynamical-mean-field-theory calculations for $\delta$-plutonium ${ }^{11}$ with excellent qualitative and semi-quantitative agreement between theory and experimental data.

Furthermore, simulated TDS patterns with increasing bending of the $\mathrm{T}(111)$ branches show indeed that there is an discernible increase in intensity of the L spots in the TDS images as shown in Fig. 14. The corresponding Ewald sphere is shown in the top right of Fig. 14. The brightening of the L point with softening follows simply that the TDS intensity is inversely proportional to the phonon frequency given in equation 3 .

\section{$\delta$ Pu-Ga (111)}
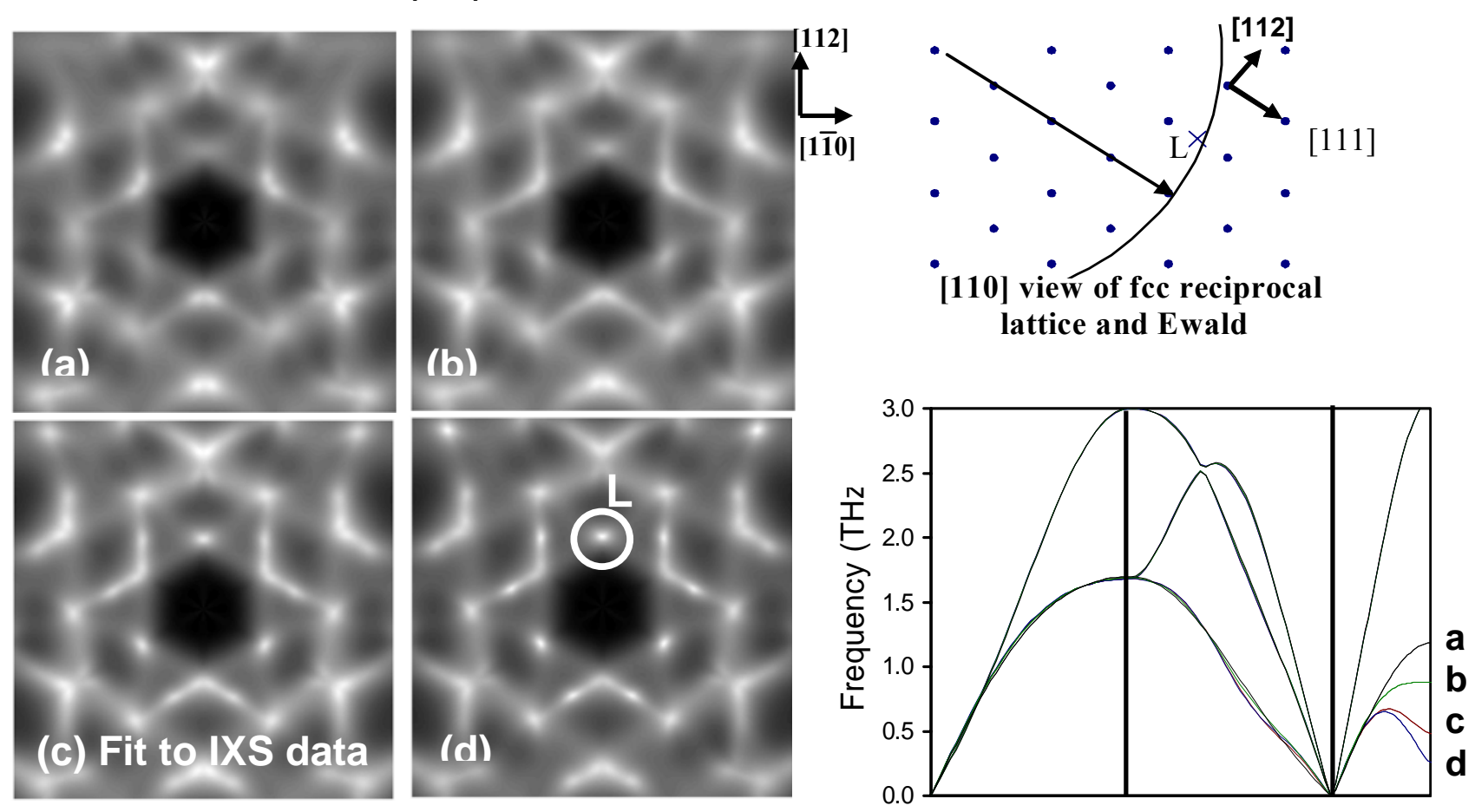

Fig. 14. (a)-(d) calculated room temperature TDS patterns of $\delta \mathrm{Pu}-\mathrm{Ga}$ (111) using the dispersion curves shown on the right (bottom) showing various bendings of the T(111) branch. Branch denoted $\mathrm{c}$ is the actual IXS data. The corresponding Ewald sphere is shown on the top right. 


$$
I_{T D S} \propto f^{2} e^{-2 M} \sum_{j=1}^{3} \frac{\left|\mathbf{q} \cdot \hat{\mathbf{e}}_{j}\right|^{2}}{\omega_{j}} \operatorname{coth}\left(\frac{\hbar \omega_{j}}{2 k_{B} T}\right)
$$

\section{References}

1. G. Lander, Science, 3011057 (2003)

2. A. M. Boring and J. L. Smith, Los Alamos Science 26, 91 (2000).

3. S. S. Hecker, Los Alamos Science 26, 290 (2000).

4. R. Jeanloz, Phys. Today 12, 44 (2000).

5. S. S. Hecker, Prog. Mater. Science, 49, 429 (2004).

6. J. T. Orme, M. E. Faiers, B .J. Ward, in the Proc. of $5^{\text {th }}$ conf, in plutonium an other actinides, ed. by H. Blank, R. Lindner, New York, North Holland Publishing Co., (1976), p. 761.

7. A. Zheludev et al., Phys. Rev. B 51, 11310 (1995)

8. Joe Wong, M. Krisch, D. Farber, F. Occelli, A. Schwartz, Tai-C. Chiang, M. Wall, C. Boro and R. $\mathrm{Xu}$, Science, 301, 1078 (2003).

9. M. E. Manley, G. H. Lander, H. Sinn, A. Alatas, W. L. Hults, R. J. McQueeney, J. L. Smith and J. Willit, Phys. Rev. B 67, 052302 (2003)

10. S. Y. Savrasov, G. Kotliar, E. Abrahams, Nature, 410, 793 (2001).

11. X. Dai, S. Y. Savrasov, G. Kotliar, A. Migliori, H. Ledletter, and E. Abrahams, Science 300, 953 (2003).

12. G. Kotliar and D. Vollhardt, Phys. Today, 57, 53 (2004)

13. J. C. Lashley, M. G. Stout, R. A. Pereyra, M.S. Blau, J. D. Embury, Scripta Mater. 44, 2815 (2001).

14. W. H. McMaster, N. Nerr del Grande, J. H. Mallett, and J. H. Hubbel, Lawrence Radiation Laboratory Report No. UCRL-50/74 sec. 2 Rev., (1969).

15. ESRF Procedures for experiments using radioactive samples on beam lines other than BM20.

16. F. Occelli, M. Krisch, P. Loubeyre, F Sette, R. Le Toullec, C. Masciovecchio, and J.-P Rueff, Phys. Rev. B 63, 4306 (2001).

17. M. D’Astuto, P. K. Mang, P. Giura, A. Shukla, P. Ghigna, A. Mirone, M. Braden, M. Greven, M. Krisch and F. Sette, Phys. Rev. Lett. 88, 167002 (2002); H. Requardt, J. E. Lorenzo, P. Monceau, R. Currat, M. Krisch, Phys. Rev. B 66, 214303 (2002).

18. M. Born, K. Huang, Dynamical Theory of Crystal Lattices. Clarendon Press, Oxford, England, (1954). 
19. W. Kohn, Phys. Rev. Lett. 2, 393 (1959).

20. E. C. Svensson, B. N. Brockhouse and J. W. Rowe, Phys. Rev., 155, 619 (1967).

21. D. H. Dutton and B. N. Brockhouse and A. P. Miiller, Can. J. Phys. 50, 2915 (1972).

22. G. P. Srivastava, The Physics of Phonons. Adam Hilger, Bristol, England, (1990).

23. Ref. 18 , p. 142

24. C. Kittel, Introduction to Solid State Physics, $3^{\text {rd }}$ Ed., John Wiley, New York (1967), p. 122

25. R. E. MacFarlane, J. A. Rayne and C. K. Jones, Phys. Lett. 20, 234 (1966)

26. R. E. Macfarlane, Phys. Lett. 18, 91 (1965)

27. E. Walker, J.Ashkenazi and M. Dacorogna, Phys. Rev. B, 24, 2254 (1981)

28. R. J. Birgeneau, J. Cordes, G. Dolling and A. D. B. Woods, Phys. Rev. 136, A1359 (1964)

29. C. Stassis, G. S. Smith, B.N. Harmon, K.-M. Ho and Y. Chen, Phys. Rev. B 31, 6298 (1985).

30. C. Stassis, T. Gould, O. D. McMasters, K. A.Gschneidner Jr., R. M. Nicklow, Phys. Rev. B 19, 5746 (1979); C. Stassis, C.-K. Loong, O.D. McMasters, R. M. Nicklow, Phys. Rev. B 25, 6485 (1982).

31. J. A. Rayne, Phys. Rev. 118, 1545 (1960)

32. J. W. Lynn, H. G. Smith and R. M. Nicklow, Phys. Rev. B 8, 3493 (1973).

33. W. Drexel, Z. Phys. 205, 281 (1972).

34. W. C. Overton, Jr., J. Gaffney, Phys. Rev. 98, 969 (1955)

35. C. Stassis, J. Zaretsky, D. K. Misemer, H. L. Skriver, and B. N. Harmon, Phys. Rev. B 27, 3303 (1983).

36. P. E. Armstrong, O. N. Carlason and J. F. Smith, J. Appl. Phys. 30, 36 (1959).

37. R. A. Reese, S. K., Sinha and D. T. Peterson, Phys. Rev. B 8, 1332 (1973)

38. U. Buchenau, M. Heiroth, H. R. Schober, J. Evers and G. Oehlinger, Phys. Rev. B 30, 3502 (1984)

39. D. L. Waldorf, Bull. Am. Phys. Soc. 5, 170 (1960).

40. B. N. Brockhouse, T. Arase, G. Caglioti, K. Rao, and A. D. Woods, Phys. Rev. 128, 1099 (1962).

41. C. Stassis, C.-K. Loong, J. Zarestky, Phys. Rev. B 26, 5426 (1982).

42. C. Stassis, C.-K. Loong, and C. Theisen, Phys. Rev. B 26, 4106 (1982)

43. J. Zarestky and C. Stasis, Phys. Rev. B 35, 4500 (1987).

44. H. M. Ledbetter, R.L. Moment, Acta Metallurgica 24, 891 (1976).

45. C. Zener, Elasticity and Anelasticity of Metal,. Univ. Chicago Press. Chicago, IL (1948), p. 16

46. P. Soderlind, A. Landa, B. Sadigh, L. Vitos and A. Ruban, Phys. Rev. B 70, 144103 (2004)

47. A. P. Miiller and B.N. Brockhouse, Can. J. Phys. 49, 704 (1971).

48. A. P. Miiller and B.N. Brockhouse, Phys. Rev. Lett, 20798 (1968). 
49. R. J. McQueeney, A. C. Lawson, A. Migliori, T. M. Kelley, B. Fultz, M. Ramos, B. Martinez, J. C. Lashley, and Sven C. Vogel, Phys. Rev. Lett. 92, 146401 (2004)

50. J. C. Lashley, J. Singleton, A. Migliori, J. B. Betts, R. A . Fisher, J. L. Smith and R. J. McQueeney, Phys. Rev. Lett. 91, 205901 (2003).

51. E. J. Nelson, K. J .M. Blobaum, M. A. Wall, P. G. Allen, A. J. Schwartz and C. H. Booth, Phys. Rev. B 67, 224206 (2003).

52. S. S.Hecker, L. F. Timofeeva, Los Alamos Sci, 26, 244 (2000)

53. W. H. Zachariasen, F. H. Ellinger, Acta Cryst. 16, 777 (1963).

54. W. H. Zachariasen, F. H. Ellinger, Acta Cryst. 8, 431 (1955)

55. P. H. Alder, G. B. Olson, and D. S. Margolies, Acta Metall. 34, 2053 (1986)

56. Y. M. Jin, Y .U. Wang, A .G. Khachaturyan, C. R. Krenn, A. J. Schwartz, Metall. Mater. Trans. A. (2004) submitted

57. T. G. Zocco, M. F. Stevens, P. H. Alder, R. T. Sheldon and G .B. Olson, Acta Metall. Mater. 38, 2275 (1990).

58. R. W. K. Honeycombe, Steels: Microstructure and Properties, Edward Arnold Publ. London (1980), p. 81

59. W. A. Harrison, Phys. Rev. B 69, 113106 (2004). 


\section{Postscript by one of us (JW)}

For an experimentalist, the Pu phonon project is a rare lifetime experience. The opportunity to seek basic knowledge and do science on this unique material is both a privilege and a responsibility. It is a privilege because of the fact that the material is available in only less than a couple of handfuls of institutions in the country (and in the globe for that matter). This is to be contrasted with Pu theoretical work which can be performed anywhere, for instance at a university or any institution. Basically, one needs a good (and realistic) potential and fast computers. It is also a responsibility because of the nature of the material, its health related hazard, environmental security, and so on.

Upon deeper reflection, Pu experiments are also very fragile! The fragility can be realized in the experimental runs of our phonon project over the past three years (Table below). Only two of the seven IXS runs resulted in usable data and only two of the five TDS runs yielded analyzable data. The five non-productive IXS runs were brought about by institutional policy (no Ga-variation work in $\mathrm{Pu}$ Ga alloys at ESRF), authorization paperwork hold-up (CIREA, France), poor-quality samples (LLNL) and unexpected material property at low temperature. The three non-productive TDS runs were all due to poor samples and lack of in-house characterization (LLNL). This was the first, and indeed the last time that the author of this postscript ran short of experimental samples at a storage ring facility in his $25^{+}$years of synchrotron radiation research.

Finally, it has been commented that in the absence of good experiments, theories are allowed to flourish. However, with the advent of bona-fide experimental phonon dispersion curves for $\mathrm{Pu}$, theorists have ample good reasons to produce a realistic $\mathrm{Pu}$ potential for the $\delta$-Pu and to construct a theory not only to help understand and explain the lattice dynamics of this correlated system, but also 
to be able to predict yet-unmeasured phonon properties such as the dependence on temperature and pressure.

Table 6. Summary of IXS and TDS Experimental Runs at ESRF and APS respectively

Date Experimental Run

$8 / 02$

$2 / 03$

$3 / 03 \quad$ IXS

$11 / 03 \quad$ IXS

2/04 IXS

$3 / 04$

4/04 IXS

$7 / 04$

$12 / 04$

6/05 IXS

7/05 IXS

$8 / 05$

TDS

TDS

TDS

TDS

TDS
* Imaged the first (100) and (111) TDS at RT

* Mapped 3 LA Branches

* Mapped 4 TA Branches and completed full dispersions

Cancelled, Ga variation forbidden at ESRF

Cancelled, CIREA goofed up paperwork; instead, tested

low temp IXS setup to $\pm 0.1 \mathrm{~K}$ at $200 \mathrm{~K}$ for $30^{+} \mathrm{h}$

Investigated TDS vs Ga concentration at RT:

* $0.6 \mathrm{wt} \% \mathrm{Ga}$ - recorded (110) Image at RT

Poor samples, 2 samples +2 empty cells to ESRF were full of Debye rings and not analyzable

$0.6 \mathrm{wt} \% \mathrm{Ga}$ : recorded (110) and (111) images at RT and $200 \mathrm{~K}$, but again full of Debye rings

Reproduced 111 branch bending, but sample somehow was stressed out at LT $\Rightarrow$ no LT data

Cancelled, no sample
$0.6 \mathrm{wt} \% \mathrm{Ga}$ : recorded (111) and (110) images from $300-200$ in $20 \mathrm{~K}$ steps. Images were still not analyzable. 